\title{
Symbolically Bounding the Drift in Time-Constrained MSC Graphs ${ }^{\star}$
}

\author{
S. Akshay ${ }^{1}$, Blaise Genest ${ }^{1,2}$, Loïc Hélouët ${ }^{1}$, and Shaofa Yang ${ }^{3}$ \\ 1 IRISA, INRIA Rennes - ENS Cachan Bretagne - CNRS, France \\ 2 CNRS, UMI IPAL joint with NUS and A*STAR/I2R, Singapore \\ 3 SIAT, Chinese Academy of Sciences, China \\ \{akshay,bgenest\}@irisa.fr, loic.helouet@inria.fr, sf.yang@siat.ac.cn
}

\begin{abstract}
Verifying systems involving both time and concurrency rapidly leads to undecidability, and requires restrictions to become effective. This paper addresses the emptiness problem for time-constrained MSC-Graphs (TC-MSC graphs for short), that is, checking whether there is a timed execution compatible with a TC-MSC graph specification. This problem is known to be undecidable in general [11], and decidable for some regular specifications [11]. We establish decidability of the emptiness problem under the condition that, for a given $K$, no path of the TC-MSC graph forces any node to take more than $K$ time units to complete. We prove that this condition can be effectively checked. The proofs use a novel symbolic representation for runs, where time constraints are encoded as a system of inequalities. This allows us to handle non-regular specifications and improve efficiency w.r.t. using interleaved representations.
\end{abstract}

\section{Introduction}

In a distributed system, several processes interact to implement a protocol. One way to describe these interactions is through scenarios, formalized using Message Sequence Charts (MSCs) [13]. MSCs describe finite interactions among agents that communicate asynchronously. A protocol is described by allowing choices and repetition of these MSCs. To specify these main characteristics while abstracting away details of implementation, the formal methods community often considers $M S C$ graphs, which are directed graphs whose nodes are labeled by MSCs. Protocol specifications also include timing requirements for messages as well as descriptions of how to recover from timeouts. To specify how time and concurrency influence each other, MSCs and MSC graphs have been generalized to time-constrained MSCs (TC-MSCs) and time-constrained MSC graphs (TC-MSC graphs) [2]. The timing information is captured by adding timing constraints between pairs of events, and transitions have additional timing constraints.

We consider decidability issues for TC-MSC graphs. This is a challenging task due to the presence of both time and concurrency. First, the set of executions of a TC-MSC graph is not regular in general. Even checking whether there exists a timed execution that is consistent with all the constraints of a model

\footnotetext{
* funded by the French Consulate at Guangzhou, ANR IMPRO, and the DST project.
} 
is non-trivial. This question, called the emptiness problem, is undecidable for TC-MSC graphs in general [11]. However, it is decidable for (sequential) timed automata [4]. Extending decidability results to distributed systems has been done in two particular and limited settings. In the first setting $[15,10]$, clocks are local to a process, and so, one cannot specify time taken by a communication (message or synchronization). This limitation makes the specification formalism very weak. The second setting can relate clocks from different processes and specify how long a communication takes, but the specifications can only exhibit regular behaviors $[2,3,7,8,18]$, which is a significant restriction in a concurrent setting where even the simple producer-consumer protocol is not regular. To obtain regularity (and hence decidability), these papers restrict the concurrency in a structural way, for instance considering only locally synchronized (see $[16,5,12]$ ) MSC graphs (in $[2$, $3]$ ) or only safe Petri Nets (in $[7,8]$ ). In [1], the language is restricted to being representable by a regular set, using both $K$-drift-boundedness - that we use in this paper and define below - and a restriction on Zeno behaviors. Decidability of checking $K$-drift-boundedness was however left open. Last, the procedures for TC-MSC graphs in $[2,3,11,1]$ construct an interleaved timed automaton, leading to a combinatorial explosion. This could be seen as going against the spirit of MSCs, which try to avoid interleavings. Further, the approaches in $[2,3,11,18,1]$ add another blow-up in complexity through the use of zone construction [4].

In this paper, we prove a novel decidability result for timed concurrent systems with global clocks having a possibly non-regular set of behaviors. We investigate the emptiness problem for TC-MSC graphs, and prove it to be decidable in the setting where a TC-MSC graph is prohibited from forcing any TC-MSC appearing along one of its paths to take an arbitrarily long amount of time to complete. More precisely, for a given integer $K$, for any path $\rho$ of a TC-MSC graph, if there exists at least one execution of $\rho$, then we require that there exists one in which the occurrence times of any two events from the same TC-MSC differ by at most $K$. Such a TC-MSC graph is said to be $K$-drift-bounded [1]. We further show that given $K$, one can effectively test whether a TC-MSC graph $G$ is $K$-drift-bounded. Both results are established without constructing an interleaved timed automaton or relying on the seminal result on decidability of emptiness of timed automata [4], avoiding both state space explosions. Instead, we translate the set of time constraints of a path into a symbolic profile, in the form of a system of inequalities. We show how to manipulate this system symbolically using Fourier-Motzkin elimination [9]. We approximate symbolic profiles by a bounded system of inequalities whose coefficients are integers in $\left[-K^{\prime}, K^{\prime}\right]$ for some integer $K^{\prime}$ depending on $G$ and $K$. This does not hinder checking consistency of $K$-driftbounded TC-MSC graphs. This forms the cornerstone of our decidability results, as finite state automata can keep track of bounded systems of inequalities.

The paper is organized as follows: Section 2 recalls basic definitions. Section 3 discusses drift-boundedness and its relevance. Section 4 shows how to check emptiness for $K$-drift-bounded TC-MSC graphs and Section 5 shows that checking $K$-drift-boundedness is decidable, for a given $K$. Omitted proofs are available in the appendix. 


\section{Preliminaries}

Let $\mathbb{R}_{\geq 0}$ denote the set of non-negative reals, $\mathbb{N}$ the set of integers and $\mathcal{I}$ the collection of open and closed intervals with end points in $\mathbb{N}$ as well as intervals of the form $[c, \infty),(c, \infty)$, where $c \in \mathbb{N}$. Throughout this paper, we fix a finite set $\mathcal{P}$ of processes and let $p, q$ range over $\mathcal{P}$. Let $\Sigma=\{p ! q, p ? q \mid p, q \in \mathcal{P}, p \neq q\}$ be the communication alphabet. The letter $p ! q$ represents $p$ sending a message to $q$, while $p ? q$ signifies $p$ receiving a message sent by $q$. We define the map loc $: \Sigma \rightarrow \mathcal{P}$ via $l o c(p ! q)=p=\operatorname{loc}(p ? q)$, and call $l o c(a)$ the location of $a$. We define Message Sequence Charts (MSCs) and time-constrained MSCs (TC-MSCs) as usual. We do not require FIFO ordering among messages.

Definition 1. An MSC is a tuple $\left(E,\left(<_{p}\right)_{p \in \mathcal{P}}, \mu, \lambda\right)$. The set of events is $E$ and $\lambda: E \rightarrow \Sigma$ labels events with letters. For each $p,<_{p}$ is a total order over $E_{p}=\{e \in E \mid \operatorname{loc}(\lambda(e))=p\}$. The message function $\mu \subseteq E_{S} \times E_{R}$ is a bijection, such that $f=\mu(e)$ implies $\lambda(e)=p ! q, \lambda(f)=q$ ? $p$ for some $p, q \in \mathcal{P}$, with $E_{S}=\{e \in E \mid \exists p, q \in \mathcal{P}, \lambda(e)=p ! q\}$ and $E_{R}=\{f \in E \mid \exists p, q \in \mathcal{P}, \lambda(f)=q ? p\}$. We require that the transitive closure $\leq$ of $\lessdot=\bigcup_{p \in \mathcal{P}}<_{p} \cup \mu$ is a partial order.

The relation $\leq$ reflects causal ordering of events. We will write $e<f$ when $e \leq f$ and $e \neq f$. Notice that $E_{p}$ has a unique $<_{p}$-maximal event (respectively, minimal event), which we refer to as the last (respectively, first) event of $E$ on $p$.

Definition 2. A TC-MSC is a tuple $\left(E,\left(<_{p}\right)_{p \in \mathcal{P}}, \mu, \lambda, \delta\right)$ where $\left(E,\left(<_{p}\right)_{p \in \mathcal{P}}, \mu, \lambda\right)$ is an $M S C$ and $\delta$ is a function associating an interval $\delta\left(e, e^{\prime}\right) \in \mathcal{I}$ to each $e \lessdot e^{\prime}$.

For each pair of events $e \lessdot e^{\prime}$, the interval $\delta\left(e, e^{\prime}\right)$ constrains the range in which the difference between the occurrence time of $e^{\prime}$ and the occurrence time of $e$ can lie. For clarity, we shall refer to occurrence times as dates. A TC-MSC $T$ defines a collection of MSCs with dates such that the relative differences of dates fulfill the constraints asserted in $T$.

Definition 3. Let $T=\left(E,\left(<_{p}\right)_{p \in \mathcal{P}}, \mu, \lambda, \delta\right)$ be a TC-MSC. A dated MSC generated by $T$ is a tuple $\left(E,\left(<_{p}\right)_{p \in \mathcal{P}}, \mu, \lambda, d\right)$ where $d: E \rightarrow \mathbb{R}^{+}$is such that for each $e \lessdot e^{\prime}, d\left(e^{\prime}\right)-d(e)$ is in the interval $\delta\left(e, e^{\prime}\right)$.

We denote by $\mathcal{L}(T)$ the set of dated MSCs generated by $T$. To capture infinite collections of TC-MSCs, we define TC-MSC graphs as in $[2,11]$, which are finite graphs whose nodes are labeled by TC-MSCs. Each path $\rho$ of a TC-MSC graph $G$ induces a TC-MSC by concatenating TC-MSCs labeling nodes of $\rho$. Transitions of $G$ are labeled by interval constraints, one for each process, that act as constraints on the timing between the last and first event of each process in consecutive nodes of $\rho$.

Definition 4. A TC-MSC graph is a structure $G=\left(N, \mathcal{T}, \Lambda, n_{\text {in }}, N_{f_{i}}, \longrightarrow, \Delta\right)$ where $N$ is a finite non-empty set of nodes, $\mathcal{T}$ a finite set of TC-MSCs, $\Lambda: N \rightarrow \mathcal{T}$ labels each node with a TC-MSC, $n_{i n}$ is the initial node, $N_{f i}$ the set of final nodes, $\longrightarrow \subseteq N \times N$ is the transition relation, and $\Delta$ is a labeling function which associates an interval $\Delta_{p}\left(n \rightarrow n^{\prime}\right) \in \mathcal{I}$ to each transition $n \rightarrow n^{\prime}$ and each process $p$, such that $\Delta_{p}\left(n \rightarrow n^{\prime}\right)=[0, \infty)$ if $\Lambda(n)$ or $\Lambda\left(n^{\prime}\right)$ has no event on process $p$. 

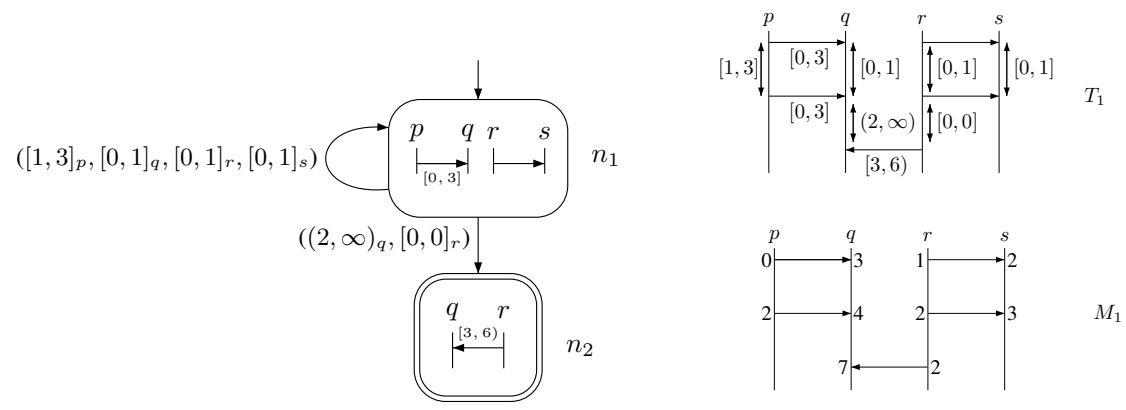

Fig. 1. A TC-MSC graph $G_{1}$, a TC-MSC $T_{1}$ and a dated MSC $M_{1} \in \mathcal{L}\left(G_{1}\right)$.

A path $\rho$ of the TC-MSC graph $G$ is a sequence $n_{0} n_{1} \ldots n_{\ell}$ such that $n_{0}=n_{\text {in }}$ and $n_{i} \rightarrow n_{i+1}$ for $i=0, \ldots, \ell-1$. The path $\rho$ is said to be final if $n_{\ell} \in N_{f i}$. For each $n \rightarrow n^{\prime}$, the concatenation of TC-MSCs $\Lambda(n), \Lambda\left(n^{\prime}\right)$ is defined with respect to $\Delta\left(n \rightarrow n^{\prime}\right)$, and is denoted $\Lambda(n) \circ \Lambda\left(n^{\prime}\right)$. Roughly speaking, this consists of putting $\Lambda\left(n^{\prime}\right)$ after $\Lambda(n)$ and for every process $p$, attaching to the pair $\left(e_{p}, f_{p}\right)$ the constraint $\Delta_{p}\left(n \rightarrow n^{\prime}\right)$, for $e_{p}$ the last event of $\Lambda(n)$ on process $p$ and $f_{p}$ the first event of $\Lambda\left(n^{\prime}\right)$ on $p$. Formally, let $\Lambda(n)=\left(E,\left(<_{p}\right)_{p \in \mathcal{P}}, \mu, \lambda, \delta\right), \Lambda\left(n^{\prime}\right)=\left(E^{\prime},\left(<_{p}^{\prime}\right.\right.$

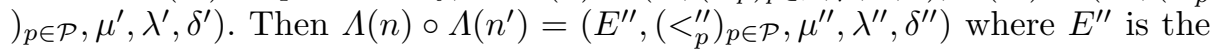
disjoint union of $E$ and $E^{\prime},<_{p}^{\prime \prime}$ is the transitive closure of the union of $<_{p},<_{p}^{\prime}$ and $E_{p} \times E_{p}^{\prime}$, and $\lambda^{\prime \prime}$ is given by: $\lambda^{\prime \prime}(e)=\lambda(e)$ for $e \in E, \lambda^{\prime \prime}(e)=\lambda^{\prime}(e)$ for $e \in E^{\prime}$. We also set $\mu^{\prime \prime}(e)=\mu(e)$ when $\mu(e)$ is defined, and $\mu^{\prime \prime}(e)=\mu^{\prime}(e)$ when $\mu^{\prime}(e)$ is defined. At last, $\delta^{\prime \prime}$ is given by: $\delta^{\prime \prime}(e, f)=\delta(e, f)$ for $e \lessdot f, \delta^{\prime \prime}(e, f)=\delta^{\prime}(e, f)$ for $e \lessdot^{\prime} f$. For each $p$, if both $E_{p}$ and $E_{p}^{\prime}$ are nonempty, we set $\delta^{\prime \prime}\left(e_{p}, f_{p}\right)=\Delta_{p}\left(n \rightarrow n^{\prime}\right)$ for $e_{p}$ the last event of $E_{p}$ and $f_{p}$ the first event of $E_{p}^{\prime}$.

We emphasize that by definition, $\Delta_{p}\left(n \rightarrow n^{\prime}\right)=[0, \infty)$ if $E_{p}$ or $E_{p}^{\prime}$ is empty. It follows that for $n \rightarrow n^{\prime} \rightarrow n^{\prime \prime},\left(\Lambda(n) \circ \Lambda\left(n^{\prime}\right)\right) \circ \Lambda\left(n^{\prime \prime}\right)$ is the same as $\Lambda(n) \circ\left(\Lambda\left(n^{\prime}\right) \circ\right.$ $\left.\Lambda\left(n^{\prime \prime}\right)\right)$. Thus, we unambiguously define the TC-MSC $T^{\rho}$ induced by a path $\rho=n_{0} \ldots n_{\ell}$ of $G$ to be $\Lambda\left(n_{0}\right) \circ \ldots \circ \Lambda\left(n_{\ell}\right)$. A path $\rho$ of $G$ is called consistent if $\mathcal{L}\left(T^{\rho}\right) \neq \emptyset$. From now on, we will speak interchangeably of a node $n$ and its associated TC-MSC $\Lambda(n)$. We write $\mathcal{L}(G)$ for the union of $\mathcal{L}\left(T^{\rho}\right), \rho$ ranging over final paths of $G$. We call a dated MSC in $\mathcal{L}(G)$ a timed execution of $G$. An example of a TC-MSC graph $G_{1}$ is in Figure 1 . The TC-MSC $T_{1}$ is induced by path $n_{1} \cdot n_{1} \cdot n_{2}$ of $G_{1}$, i.e., $T_{1}=T^{n_{1} \cdot n_{1} \cdot n_{2}}$. Further, $M_{1}$ is a dated MSC generated by $T_{1}$. As $n_{2}$ is final, $M_{1} \in \mathcal{L}\left(G_{1}\right)$.

The emptiness problem for TC-MSC graphs is: given a TC-MSC graph $G$, determine whether $\mathcal{L}(G)=\emptyset$, that is, whether it has no consistent and final path. This is a fundamental verification problem that must be addressed. Indeed, a TC-MSC graph with an empty language should be considered ill-specified and such an exception should be caught at an early stage of design. In [11], it is shown that this problem is undecidable in general, and decidable for some regular specifications. We show in the following that checking emptiness for TC-MSC graphs is decidable under an arguably mild restriction on time constraints which does not impose regularity. Furthermore, we will show that one can test whether a given TC-MSC graph satisfies this condition. 


\section{Drift-Boundedness}

In this section we define our mild restriction, namely drift-boundedness. Let us fix a TC-MSC graph $G$. Let $\rho=n_{0} \ldots n_{\ell}$ be a consistent path of $G$ and $M=\left(E,\left(<_{p}\right)_{p \in \mathcal{P}}, \mu, \lambda, d\right)$ be a dated MSC generated by $T^{\rho}$. For an integer $K$, we say that $M$ is a $K$-drift-bounded dated MSC of $\rho$ iff for each $i=0, \ldots, \ell$, for any two events $e, e^{\prime}$ in $\Lambda\left(n_{i}\right)$, it is the case that $\left|d(e)-d\left(e^{\prime}\right)\right| \leq K$. We say that $\rho$ is $K$-drift-bounded iff there exists a $K$-drift-bounded dated MSC in $\mathcal{L}\left(T^{\rho}\right)$. We emphasize that $\mathcal{L}\left(T^{\rho}\right)$ may also contain dated MSCs which are not $K$-drift-bounded. We say that $G$ is $K$-drift-bounded iff every consistent (but not necessarily final) path of $G$ is $K$-drift-bounded. In other words, for each consistent path $\rho$, we can find a dated MSC in $\mathcal{L}\left(T^{\rho}\right)$ such that the difference between the dates of any two events from the same instance of a node is at most $K$. Notice that we can have $\mathcal{L}(G)=\emptyset$ even though $G$ is $K$-drift-bounded. In fact, $G$ is vacuously $K$-drift-bounded for any $K$ if it has no consistent path.

As an example, consider the TC-MSC graph $G_{1}$ from Figure 1. $G_{1}$ is 3 -driftbounded since in every timed execution, we can be sure that all events in node $n_{1}$ or $n_{2}$ can be completed within a delay of 3 time units. But if we change the constraints on the loop on $n_{1}$ from $\left([0,1]_{r},[0,1]_{s}\right)$ to $\left([4,5]_{r},[1,2]_{s}\right)$ then for any integer $K, G_{1}$ is not $K$-drift-bounded. Note that $G_{1}$ is not locally synchronized (as defined in $[16,5]$, and lifted in [3] to a timed setting). In fact, we can simulate the producer-consumer protocol and obtain non-regular behaviors. Thus, this example cannot be handled by the decidability result in [3].

We believe that drift-boundedness is a practical notion. Interpreting a node of a TC-MSC graph as a phase or a transaction of a distributed protocol, we expect any scenario labeling the node to be executable in a bounded time, say $K$. A protocol specified as a TC-MSC graph that is not $K$-drift-bounded should thus be considered as ill-formed. Indeed, while a TC-MSC graph specification is usually incomplete (as it abstracts away some events and constraints used in the actual implementation), if it is not $K$-drift-bounded, then every implementation of this specification will not be $K$-drift-bounded either.

\subsection{The main results}

We can now state our main results. The first result establishes the decidability of the emptiness problem for $K$-drift-bounded TC-MSC graphs.

Theorem 1. Let $K \in \mathbb{N}$ and $G$ be a $K$-drift-bounded TC-MSC graph. Then checking whether $\mathcal{L}(G)$ is empty is decidable in PSPACE.

We next show that the drift-boundedness hypothesis of Theorem 1 can be effectively checked, giving rise to an effective decidability procedure.

Theorem 2. Let $K \in \mathbb{N}$ and $G$ be a $T C$-MSC graph. Then checking whether $G$ is $K$-drift-bounded is decidable in PSPACE.

We can show that the decidability result in Theorem 2 is in fact at the boundary of undecidability. Recall that the definition of $K$-drift-bounded considers 
every path of a TC-MSC graph, including paths that cannot be extended to consistent final paths. Instead, if we consider the problem of checking whether every consistent final path of a TC-MSC graph is $K$-drift-bounded, this turns out to be undecidable. We assume $K$ fixed for the next proposition.

Proposition 1. It is undecidable, given a TC-MSC graph $G$, to determine whether every consistent final path of $G$ is $K$-drift-bounded.

Proof. The proof is by a reduction from the emptiness problem of TC-MSC graphs, shown undecidable in [11]. Let $G$ be a TC-MSC graph. We construct another TC-MSC graph $G^{\prime}$ from $G$ such that there does not exist a consistent final path of $G$ iff every consistent final path of $G^{\prime}$ is $K$-drift-bounded, which shows the result. $G^{\prime}$ is obtained from $G$ with the following modifications. Firstly, add a new node $n_{\text {new }}$ and for every final state $n_{f}$ of $G$, add a transition $\left(n_{f}, n_{\text {new }}\right)$. Secondly, define the set of final nodes of $G^{\prime}$ to be the singleton set $\left\{n_{n e w}\right\}$. Thirdly, $n_{n e w}$ is labeled with a TC-MSC consisting of a single message $(e, f)$ from $p$ to $q$. The time constraint on $(e, f)$ is $[K+1, K+1]$. Lastly, for every final state $n_{f}$ of $G$ and every process, the time constraint of transition $\left(n_{f}, n_{\text {new }}\right)$ is $[0, \infty)$. If there does not exist a consistent final path of $G$, then there does not exist a consistent final path of $G^{\prime}$, and it is vacuously true that every consistent final path of $G^{\prime}$ is $K$-drift-bounded. On the other hand, assume that there exists some consistent final path $\rho$ of $G$. Then $\rho \cdot n_{\text {new }}$ is a consistent final path of $G^{\prime}$ (timing of a consistent dated MSC of $\rho$ can be easily extended). But it is not $K$-drift-bounded because of the constraint $[K+1, K+1]$ on the last node $n_{\text {new }}$ of the path, which impose $e, f$ to be $K+1$ time units away. Hence not every consistent final path of $G^{\prime}$ is $K$-drift-bounded.

Next, we introduce full TC-MSC graphs and show that any TC-MSC graph can be transformed into a full TC-MSC graph, while preserving consistency and drift-boundedness of paths. This enables us to check both the emptiness of a $K$-drift-bounded TC-MSC graph $G$, and the $K$-drift-boundedness of any TC-MSC graph $G$, by working with a full TC-MSC graph constructed from $G$.

\section{$3.2 \quad$ Full TC-MSC Graphs}

We call a TC-MSC graph $G$ full if each node of $G$ has at least one event on each process $p \in \mathcal{P}$. We will now show how to "augment" a TC-MSC graph $G$ to obtain a full TC-MSC graph $\widehat{G}$ by adding "dummy events" to nodes of $G$. For notational convenience, we assume that TC-MSCs may contain internal events. We denote by $p($ int $)$ the label of such an internal event on process $p \in \mathcal{P}$.

Given $G=\left(N, \mathcal{T}, \Lambda, n_{i n}, N_{f i}, \longrightarrow, \Delta\right)$, the augmented graph of $G$ is defined as $\widehat{G}=\left(N, \widehat{\mathcal{T}}, \widehat{\Lambda}, n_{i n}, N_{f i}, \longrightarrow, \Delta\right)$ differing only in the labeling set of "augmented" TC-MSCs and the labeling function assigning nodes to them. More precisely, any TC-MSC $T=\left(E,\left(<_{p}\right)_{p \in \mathcal{P}}, \mu, \lambda, \delta\right)$ in $\mathcal{T}$ is replaced by the TC-MSC $\widehat{T}=$ $\left(E^{\prime},\left(<_{p}\right)_{p \in \mathcal{P}}, \mu, \lambda^{\prime}, \delta\right)$ in $\widehat{\mathcal{T}}$ where $E^{\prime}$ is obtained from $E$ by adding a new event $e_{p}$ with $\lambda\left(e_{p}\right)=p($ int $)$ for each process $p$ such that $E_{p}=\emptyset$. Every $<_{p}$ and $\delta$ are 
unchanged, so $e_{p}$ is an isolated point in the partial order $\leq$. Such events $e_{p}$ will be called dummy events. Events already in $\Lambda(n)$ will be called concrete events. Note that $\Delta(n \rightarrow m)$ is unchanged for each transition $n \rightarrow m$. In particular, recall that for each transition $(n, m)$ in $G$, if either $n$ or $m$ has no concrete event on $p$, then $\Delta_{p}(n, m)=[0, \infty)$. For each $\Lambda(n)=T$, we set $\widehat{\Lambda}(n)=\widehat{T}$. Obviously, $\widehat{G}$ is full for any $G$.

Let $H$ be any full TC-MSC graph with events partitioned as dummy or concrete. That is, in every TC-MSC labeling a node of $H$, there is a mapping from the set of events to $\{d u m m y$, concrete $\}$. For instance, $\widehat{G}$ is such a full TC-MSC graph. Let $Y \leq Y^{\prime} \in \mathbb{N}$. Now, for a path $\rho=n_{0} \ldots n_{\ell}$ of $H$, we say that a dated MSC $M=\left(E,\left(<_{p}\right)_{p \in \mathcal{P}}, \mu, \lambda, d\right)$ generated by $T^{\rho}$ is $\left(Y, Y^{\prime}\right)$-drift-bounded if for each $i=0, \ldots, \ell$, for any two events $e, f$ in the TC-MSC $\Lambda\left(n_{i}\right)$, we have: (i) if both $e$ and $f$ are concrete events, then $|d(e)-d(f)| \leq Y$; (ii) if one or both of $e, f$ are dummy events, then $|d(e)-d(f)| \leq Y^{\prime}$. We say that a consistent path $\rho$ of $H$ is $\left(Y, Y^{\prime}\right)$-drift-bounded if there exists a $\left(Y, Y^{\prime}\right)$-drift-bounded dated MSC generated by $\rho$. At last, $H$ is $\left(Y, Y^{\prime}\right)$-drift-bounded if all its consistent paths are.

Proposition 2. For a TC-MSC graph $G$, a path $\rho$ of $G$ and $K \in \mathbb{N}$, (i) $\rho$ is consistent in $G$ iff $\widehat{\rho}$ is consistent in $\widehat{G}$, (ii) $\rho$ is $K$-drift-bounded in $G$ iff $\widehat{\rho}$ is $(K, \widehat{K})$-drift-bounded in $\widehat{G}$, with $\widehat{K}=(|\mathcal{P}|-1) \cdot K$.

Hence, we are able to restrict to full TC-MSC graphs when checking for emptiness using (i), and when checking for $K$-drift boundedness using (ii):

Corollary 1. Given a TC-MSC graph $G$, (i) $\mathcal{L}(G) \neq \emptyset$ iff $\mathcal{L}(\widehat{G}) \neq \emptyset$, and (ii) $G$ is $K$-drift-bounded iff $\widehat{G}$ is $(K, \widehat{K})$-drift-bounded, where $\widehat{K}=(|\mathcal{P}|-1) \cdot K$.

\section{Emptiness for $\boldsymbol{K}$-Drift-Bounded TC-MSC Graphs}

We now prove Theorem 1 . We assume $G$ to be a $K$-drift-bounded TC-MSC graph. By Corollary 1 , we can build $\widehat{G}$, a $(K, \widehat{K})$-drift-bounded full TC-MSC graph with $\mathcal{L}(\widehat{G}) \neq \emptyset$ iff $\mathcal{L}(G) \neq \emptyset$. It then suffices to check the emptiness of a finite automaton that accepts the set of $(K, \widehat{K})$-drift-bounded final paths of $\widehat{G}$.

Let $H$ be a full TC-MSC graph, with events partitioned as dummy or concrete. To avoid clutter, we assume that constraints in $H$ are only of the form $[a, b]$ and $[a, \infty)$. Extending proofs to handle other constraints is straightforward and all statements hold in general, but additional notations are needed to remember whether each inequality is strict or not. We first describe intuitively the key ingredients of the proof, which will be developed in the rest of this section.

- First, we observe that checking consistency of a path $\rho$ of $H$, i.e., $\mathcal{L}\left(T^{\rho}\right) \neq \emptyset$, is equivalent to checking for the existence of a solution to a system of inequalities over (real-valued) variables $x_{e}$ depicting the dates of events $e$ of $T^{\rho}$.

- Next, we show that checking whether a dated MSC can be extended by a node by assigning appropriate dates to events of this node can be done with information only on the relative difference of dates of the last event of the 
dated MSC on each process. This motivates us to associate a symbolic profile $P F(\rho)$ to each path $\rho$. A symbolic profile is a system of inequalities whose solutions correspond to the dates of final events of dated MSCs generated by $T^{\rho}$, and vice versa. In particular, $P F(\rho)$ has a solution iff $\rho$ is consistent.

- We remark that constants appearing in symbolic profiles can be chosen as integers. Restricting constants to be within $[-\widehat{K}, \widehat{K}]$ does not exclude any consistent $(K, \widehat{K})$-drift-bounded path of $H$. We can then represent with a finite automaton the set of consistent $(K, \widehat{K})$-drift-bounded paths of $H$.

Systems of inequalities and Fourier-Motzkin elimination. We first fix basic terminologies for systems of difference inequalities. Let $X$ be a finite nonempty set of real-valued variables. A (difference) inequality is an inequality of the form $x-y \leq a$, where $x, y$ are two different variables in $X$.

Definition 5. A system of (difference) inequalities $\phi$ over $X$ is $\wedge_{(x, y) \in R} x-y \leq$ $a_{x y}$ where $R \subseteq X \times X$ is an irreflexive relation. We say that $\phi$ has integral coefficients whenever $a_{x y}$ is a (possibly negative) integer for all $(x, y) \in R$.

From now on, we assume that the system is simplified, that is, for each $x, y \in X$, there is at most one inequality of the form $x-y \leq a$. This involves no loss of generality as $x-y \leq a \wedge x-y \leq a^{\prime}$ is equivalent with $x-y \leq \min \left(a, a^{\prime}\right)$. If $x-y \leq a$ appears in $\phi$, we say that $\phi$ contains an edge $(x, y)$, and the weight of this edge is $a$. We say that two systems $\phi, \psi$ of inequalities are equivalent when $\phi$ has a solution (in the real domain) iff $\psi$ has a solution (in the real domain).

A key idea is to propagate constraints concerning variables in a subset $Y \subsetneq X$ on variables in $X \backslash Y$, and then safely remove variables in $Y$ while keeping an equivalent system. This is done using the Fourier-Motzkin elimination method (see extended version, or $[9,14]$ ).

For $F \subseteq X$, let $\phi_{\mid F}$ denote the (unique) system of inequalities over variables $F$ obtained by performing Fourier-Motzkin elimination of variables in $X \backslash F$ following a fixed order. We have that $\phi$ and $\phi_{\mid F}$ are equivalent. If $\phi$ has integral coefficients, then so does $\phi_{\mid F}$.

Symbolic Profiles. Let $T^{\rho}=\left(E,\left(<_{p}\right), \mu, \lambda, \delta\right)$ be the TC-MSC associated with some path $\rho=n_{0} \ldots n_{\ell}$ of $H$. We denote by $x_{e}$ a $\mathbb{R}_{\geq 0}$-valued variable, standing for the date of event $e \in E$, and let $X_{E}=\left\{x_{e} \mid e \in E\right\}$. We associate path $\rho$ with a system of linear inequalities $\Phi(\rho)$ with integral coefficients as follows:

Definition 6. The system $\Phi(\rho)$ associated with $\rho$ is the smallest system of inequalities over the set of variables $X_{E}$

such that, for any $e, f \in E$ with $e \lessdot f$,

- if $\delta(e, f)=[L, U]$, then $\Phi(\rho)$ contains both $x_{f}-x_{e} \leq U$ and $x_{e}-x_{f} \leq-L$;

- if $\delta(e, f)=[L, \infty)$, then $\Phi(\rho)$ contains $x_{e}-x_{f} \leq-L$.

We easily have that $\rho$ is consistent iff $\Phi(\rho)$ has a solution. Let $e_{p}$ be the last event of $T^{\rho}$ on $p$, for each process $p$. Let $E_{\text {last }}$ be the set $\left\{e_{p} \mid p \in \mathcal{P}\right\}$. 


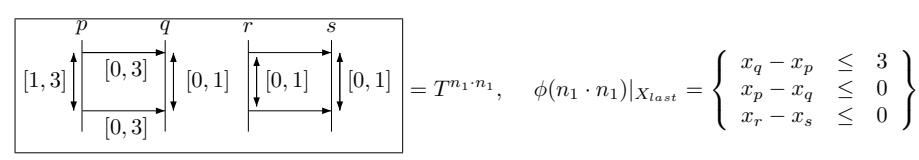

Fig. 2. The TC-MSC induced by path $n_{1} \cdot n_{1}$ of $G_{1}$ and its profile

Using Fourier-Motzkin elimination of variables $X^{\prime}=\left\{x_{e} \mid e \notin E_{\text {last }}\right\}$, we obtain a system $\left.\Phi(\rho)\right|_{X_{\text {last }}}$ over variables $X_{\text {last }}=\left\{x_{e} \mid e \in E_{\text {last }}\right\}$, with integral coefficients, equivalent with $\Phi(\rho)$. Once simplified, this system has at most $|\mathcal{P}|^{2}$ inequalities with integral coefficients. We encode this system as a symbolic profile.

Definition 7. $A$ symbolic profile $\sigma$ is a function from $\mathcal{P} \times \mathcal{P}$ to $\mathbb{Z} \cup\{\infty\}$. We denote by $\mathcal{P} \mathcal{F}$ the (infinite) set of all profiles.

Notice that symbolic profiles are syntactically similar to Difference Bounded Matrices (DBMs) [6] over $|\mathcal{P}|$ clocks. However, unlike a DBM, a symbolic profile may not correspond to a timed linearization, and the update function (defined below) is very different when compared to DBMs.

Let $\phi$ be a system of inequalities with integral coefficients over $X_{\text {last }}=\left\{x_{p} \mid\right.$ $p \in \mathcal{P}\}$. We define the symbolic profile $P F(\phi)$ induced by $\phi$ as $P F(\phi)[p, q]=a_{p q}$ if $x_{p}-x_{q} \leq a_{p q}$ belongs to $\phi$, and $P F(\phi)[p, q]=\infty$ otherwise. Intuitively, $P F(\phi)[p, q]=\infty$ means that there is no inequality of the form $x_{p}-x_{q} \leq a_{p q}$ in $\phi$. We abusively use $P F(\phi)$ as a system of inequalities in the following, and denote $x_{p}$ for $x_{e_{p}}$. For a path $\rho$, we denote $P F(\rho)=P F\left(\left.(\Phi(\rho))\right|_{X_{\text {last }}}\right)$. We say that a symbolic profile $\sigma \in \mathcal{P F}$ is satisfiable if it has a solution. It is easy to check whether $P F(\rho)$ is satisfiable, either by using Fourier-Motzkin elimination till reaching a trivial equation, or by using Shostak characterisation [17].

Proposition 3. $P F(\rho)$ is satisfiable iff $\rho$ is consistent.

As an example, consider the TC-MSC $T^{n_{1} \cdot n_{1}}$ in Figure 2, generated by path $n_{1} \cdot n_{1}$ of $G_{1}$ from Figure 1. Let $e_{j}^{i}$ denote the $i^{t h}$ event on process $j$ and $E$ be the set of events of $T^{n_{1} \cdot n_{1}}$. We obtain $\Phi\left(n_{1} \cdot n_{1}\right)$ to be the set of inequalities over $X=$ $\left\{x_{e} \mid e \in E\right\}$, where for instance the inequations $x_{e_{p}^{2}}-x_{e_{p}^{1}} \leq 3$ and $x_{e_{p}^{1}}-x_{e_{p}^{2}} \leq-1$ capture the timing constraint $[1,3]$ between $e_{p}^{1}$ and $e_{p}^{2}$. Now eliminating variables $x_{e_{p}^{1}}, x_{e_{q}^{1}}, x_{e_{r}^{1}}, x_{e_{s}^{1}}$ results in a set of equations on $X_{\text {last }}=\left\{x_{e_{p}^{2}}, x_{e_{q}^{2}}, x_{e_{r}^{2}}, x_{e_{s}^{2}}\right\}=$ $\left\{x_{p}, x_{q}, x_{r}, x_{s}\right\}$ as shown. E.g., $\left.P F\left(n_{1} \cdot n_{1}\right)\right)[p, q]=\min (3,-1+3+1)=3$ and $\left.\operatorname{PF}\left(n_{1} \cdot n_{1}\right)\right)[s, r]=\infty$. This system of inequalities has many solutions.

Bounded profiles. Notice that the set of symbolic profiles as defined above is not finite in general (the coefficients range over $\mathbb{Z}$ ), and so, it cannot be recorded by a finite state automaton. Instead, we use the finite set of $L$-bounded profiles, where $L \in \mathbb{N}$ is some integer.

Definition 8. For $L \in \mathbb{N}$, a $L$-bounded profile $\sigma$ is a function from $\mathcal{P} \times \mathcal{P}$ to $\mathbb{Z} \cap[-L, L]$. We denote by $\mathcal{P F}_{L}$ the set of $L$-bounded profiles. 
Let $Y \leq Y^{\prime} \in \mathbb{N}$. Notice that the set $\mathcal{P} \mathcal{F}_{Y^{\prime}}$ is finite. We denote by $\Phi_{Y, Y^{\prime}}(\rho)$ the system of inequalities obtained from $\Phi(\rho)$ by the following modification: for each $i=0, \ldots, \ell$, for any two different events $e, f$ in the same node $n$ of $\rho$, if $\Phi(\rho)$ contains $x_{e}-x_{f} \leq a_{e, f}$, then replace it by $x_{e}-x_{f} \leq \min \left(a_{e, f}, Y\right)$ if both $e, f$ are concrete, and by $x_{e}-x_{f} \leq \min \left(a_{e, f}, Y^{\prime}\right)$ otherwise (that is if at least one of $e$ or $f$ is dummy); if $\Phi(\rho)$ does not have an edge $(e, f)$, then add the inequality $x_{e}-x_{f} \leq Y$ if both $e, f$ are concrete, and $x_{e}-x_{f} \leq Y^{\prime}$ otherwise. Clearly, $\rho$ is consistent and $\left(Y, Y^{\prime}\right)$-drift-bounded iff $\Phi_{Y, Y^{\prime}}(\rho)$ has a solution. If $\Phi_{Y, Y^{\prime}}(\rho)$ has a solution, we set $P F_{Y, Y^{\prime}}(\rho)=P F\left(\left.\Phi_{Y, Y^{\prime}}(\rho)\right|_{X_{\text {last }}}\right)$. In a full TC-MSC graph $H$, by definition of $\Phi_{Y, Y^{\prime}}(\rho)$, we have $P F_{Y, Y^{\prime}}(\rho) \in \mathcal{P} \mathcal{F}_{Y^{\prime}}$. If $\Phi_{Y, Y^{\prime}}(\rho)$ has no solution, it is possible that $\operatorname{PF}\left(\left.\Phi_{Y, Y^{\prime}}(\rho)\right|_{X_{\text {last }}}\right) \notin \mathcal{P} \mathcal{F}_{Y^{\prime}}$. In this case, we set $P F_{Y, Y^{\prime}}(\rho)$ to be a particular profile $\perp \in \mathcal{P F}_{Y^{\prime}}$ without solution, e.g. $\perp[p, q]=0, \perp[q, p]=-1$ (which would require $1 \leq x_{p}-x_{q} \leq 0$ ).

Proposition 4. Let $\rho$ be a path of a full TC-MSC graph H. Then $P F_{Y, Y^{\prime}}(\rho) \in$ $\mathcal{P} \mathcal{F}_{Y^{\prime}}$, and $P F_{Y, Y^{\prime}}(\rho)$ is satisfiable iff $\rho$ is consistent and $\left(Y, Y^{\prime}\right)$-drift-bounded.

Notice that $P F_{Y, Y^{\prime}}(\rho)$ cannot be obtained from $P F(\rho)$. An intuitive (but wrong) idea would be to set $P F_{Y, Y^{\prime}}(\rho)[p, q]=Y^{\prime}$ for all $P F(\rho)[p, q]>Y^{\prime}$ and else $P F_{Y, Y^{\prime}}(\rho)[p, q]=P F(\rho)[p, q]$. However, setting $P F_{Y, Y^{\prime}}(\rho)[p, q]=Y^{\prime}$ for all $P F(\rho)[p, q]>Y^{\prime}$ only constrains the dates of the last events on each process. So, the bound $Y^{\prime}$ in $\Phi_{Y, Y^{\prime}}(\rho)$ must be imposed for every node of $\rho$, and these constraints on past nodes can have implications for the profile of $\rho$.

We now explain how to compute $P F_{Y, Y^{\prime}}(\rho)$ in an inductive way, by defining an extension function $\theta_{Y, Y^{\prime}}^{n^{-}}: \mathcal{P P}_{Y^{\prime}} \rightarrow \mathcal{P F}_{Y^{\prime}}$ for all transitions $n^{-} \rightarrow n$. For $\sigma \in \mathcal{P} \mathcal{F}_{Y^{\prime}}$ and a transition $n^{-} \rightarrow n$, we define the profile $\theta_{Y, Y^{\prime}}^{n^{-}}(\sigma)$ as follows:

- Form the system $\Psi=\psi_{\sigma} \wedge \psi_{n^{-} \rightarrow n} \wedge \psi_{n}$ over $X=\left\{x_{p} \mid p \in \mathcal{P}\right\} \cup\left\{x_{e} \mid e \in E_{n}\right\}$ $\left(x_{p}\right.$ represents the date of process $p$ in $\sigma, E_{n}$ the events of $\left.T^{n}\right)$, where:

- $\psi_{\sigma}$ consists of $x_{p}-x_{q} \leq \sigma[p, q]$ for every $p, q \in \mathcal{P}$, such that $\sigma[p, q] \neq \infty$.

- $\psi_{n^{-} \rightarrow n}$ contains, for each $p$ with $\Delta_{p}\left(n^{-} \rightarrow n\right)=[L, U]$, two inequalities $x_{p}-x_{f_{p}} \leq-L$ and $x_{f_{p}}-x_{p} \leq U$, where $f_{p}$ is the first event of $n$ on $p$. For each $p$ with $\Delta_{p}\left(n^{-} \rightarrow n\right)=[L, \infty), \psi_{n^{-} \rightarrow n}$ contains $x_{p}-x_{f_{p}} \leq-L$.

- $\psi_{n}$ is $\Phi_{Y, Y^{\prime}}(n)$, the system associated with the singleton path $n$.

- Perform Fourier-Motzkin elimination on $\Psi$ to remove all variables but $\left\{x_{\hat{e}_{p}}\right\}_{p \in \mathcal{P}}$ where $\hat{e}_{p}$ is the last event of $\rho \cdot n$ on $p$. Denote by $\Pi$ the resulting system (after simplification) of inequalities over $\left\{x_{\hat{e}_{p}} \mid p \in \mathcal{P}\right\}$. Set $\theta_{Y, Y^{\prime}}^{n^{-}}(\sigma)=P F(\Pi)$. If at any stage of Fourier-Motzkin elimination, the system is not satisfiable, then set $\theta_{Y, Y^{\prime}}^{n^{-}} n(\sigma)$ to be the un-satisfiable profile $\perp \in \mathcal{P} \mathcal{F}_{Y^{\prime}}$.

Lemma 1. For a path $\rho$ ending in $n^{-}$and a transition $n^{-} \rightarrow n$, we have that $P F_{Y, Y^{\prime}}(\rho \cdot n)$ and $\theta_{Y, Y^{\prime}}^{n^{-}}\left(P F_{Y, Y^{\prime}}(\rho)\right)$ have the same set of solutions.

Construction of a Symbolic Automaton. We now construct a symbolic automaton $\mathcal{A}(H)$ accepting the final $\left(Y, Y^{\prime}\right)$-drift-bounded paths of $H$. 
Proposition 5. Let $H$ be a full TC-MSC graph with $|H|$ nodes. Then there exists an automaton $\mathcal{A}(H)$ with at most $|H| \times\left(2 \cdot Y^{\prime}+1\right)^{|\mathcal{P}|^{2}}$ states, such that $\mathcal{L}(\mathcal{A}(H)) \neq \emptyset$ iff $H$ has a (consistent) final $\left(Y, Y^{\prime}\right)$-drift-bounded path.

Proof (sketch). The states of $\mathcal{A}(H)$ are pairs $(n, \sigma)$, with $n$ a state of $H$ and $\sigma \in \mathcal{P} \mathcal{F}_{Y^{\prime}}$. The initial state is $\left(n_{i n}, P F_{Y, Y^{\prime}}\left(n_{i n}\right)\right)$. A state $(n, \sigma)$ is final if $n$ is final, and $\sigma$ is satisfiable. There is a transition labeled by $n^{\prime}$ from $(n, \sigma)$ to $\left(n^{\prime}, \sigma^{\prime}\right)$ iff both $\sigma, \sigma^{\prime}$ are satisfiable, there is a transition from $n$ to $n^{\prime}$, and $\sigma^{\prime}=\theta_{Y, Y^{\prime}}^{n \rightarrow n^{\prime}}(\sigma)$. The proof now follows from Lemma 1 and Proposition 4.

The proof of Theorem 1 follows from this: as every path of $\widehat{G}$ is $(K, \widehat{K})$-driftbounded, taking $H=\widehat{G}, Y=K, Y^{\prime}=\widehat{K}$ implies $\mathcal{L}(\mathcal{A}(\widehat{G})) \neq \emptyset$ iff $\mathcal{L}(\widehat{G}) \neq \emptyset$ (iff $\mathcal{L}(G) \neq \emptyset$ by Corollary 1$)$. Now, checking that $\mathcal{L}(\mathcal{A}(\widehat{G})) \neq \emptyset$ is decidable in space logarithmic in $|G|, K$ and polynomial in $|\mathcal{P}|$.

Compared with [3], which builds an automaton accepting every timed linearizations of a regular TC-MSC graph, we end up with a much smaller automaton in the worst case (exponential in $|\mathcal{P}|^{2}$ instead of exponential in $|G|$ for [3]). Further, being symbolic, we believe that the worst case is seldom reached, contrary to constructions based on zones of timed automata $[3,1,2,18]$. Indeed, consider a path $\rho$ made of one node, labeled by a TC-MSC with one event $e_{p}$ for every $p \in \mathcal{P}$, and without constraints, hence allowing events to occur at any date. Without symbolic encoding, this path would give rise to $|2 K|^{|\mathcal{P}|}$ configurations of the form $\left(x_{p}\right)_{p \in \mathcal{P}}$, with $x_{p} \in\{0,(0,1), 1, \cdots, K\}$ the clock associated with $e_{p}$. Our solution only memorizes the unique symbolic profile $P F_{K, \widehat{K}}(\rho)$ such that $\forall p, q \in \mathcal{P}, P F_{K, \widehat{K}}(\rho)[p, q]=\widehat{K}$, meaning that $-\widehat{K} \leq x_{p}-x_{q} \leq \widehat{K}$ for all $p, q$.

\section{Checking $K$-Drift-Boundedness of TC-MSC Graphs}

The construction of automaton $\mathcal{A}(\widehat{G})$ in Section 4 allows to decide the emptiness of $\mathcal{L}(\widehat{G})$ (and hence of $\mathcal{L}(G)$ ), under the hypothesis that $G$ is $K$-drift-bounded. We show here that given $K$, one can decide whether $G$ is $K$-drift-bounded. We use Proposition 2 to create a full TC-MSC graph $\widehat{G}$. The main idea is that if $\widehat{G}$ is not $(K, \widehat{K})$-drift-bounded, then there must be a path of "minimal" length which is consistent but not $(K, \widehat{K})$-drift-bounded. The idea is then to look for such a minimal witness. We call a path $\rho \cdot n$ of $\widehat{G}$ a minimal witness iff:

1. The path $\rho$ is $(K, \widehat{K})$-drift-bounded, and

2. The path $\rho \cdot n$ is not $(K, \widehat{K})$-drift-bounded, and

3. The path $\rho \cdot n$ is consistent.

Remark 1. $G$ is not $K$-drift-bounded iff $\widehat{G}$ is not $(K, \widehat{K})$-drift-bounded iff there exists a minimal witness in $\widehat{G}$.

Now we build a finite automaton recognizing exactly the set of minimal witnesses of $\widehat{G}$ which from the remark above immediately proves Theorem 2 . Requirements 1 . and 2. are easy to check with the automaton built in the previous 
section. Requirement 3. is harder to check on its own as there is no effectively constructible finite state automaton accepting all consistent paths, (since it is undecidable to know whether there exists a consistent final path [11]). However, we will prove that thanks to requirement 1., requirement 3 . can be replaced by: the path $\rho \cdot n$ is consistent and $K_{2}$-drift-bounded, for some contant $K_{2}$ depending on $G$ and $\widehat{K}$. Notice that fixing $K_{2}=\widehat{K}$ may not be enough.

The bound $K_{2}$ is chosen as follows. For a node $n$ in $\widehat{G}$, set $D_{n}$ to be the sum of lower bounds of $\delta(e, f)$, for every pair $(e, f)$ with $e \lessdot f$. For a transition $\left(n, n^{\prime}\right)$ in $\widehat{G}$, set $D_{\left(n, n^{\prime}\right)}$ to be the sum of the lower bounds of $\Delta_{p}\left(n, n^{\prime}\right)$ for $p$ ranging over $\mathcal{P}$. Set $D(\widehat{G})$ to be the maximum of $D_{\left(n, n^{\prime}\right)}+D_{n^{\prime}}$ where $\left(n, n^{\prime}\right)$ ranges over transitions of $\widehat{G}$. Finally, we let $K_{2}=(|\mathcal{P}| / 2+1) \cdot \widehat{K}+D(\widehat{G})$.

Proposition 6. Let $\rho \cdot n$ be a path of $\widehat{G}$ such that $\rho$ is $(K, \widehat{K})$-drift-bounded. Then $\rho \cdot n$ is consistent iff $\Phi_{K_{2}, K_{2}}(\rho \cdot n)$ is satisfiable.

The technical proof uses the characterization of consistent systems of equations given by Shostak lemma [17], which we explain now.

Recall that consistency of a path $\rho$ in $\widehat{G}$ is equivalent to satisfiability of the associated system of inequalities $P F(\rho)$. Let $\varphi$ be a (simplified) system of inequalities. A cycle in $\varphi$ is a sequence $x_{1} \ldots x_{m}$ such that for all $i \in\{1, \ldots, m-1\}$, $x_{i+1}-x_{i} \leq a_{i}$ appears in $\phi$ for some $a_{i}$, and $x_{m}=x_{1}$. The weight of this cycle is $\sum_{i \in\{1, \ldots, m-1\}} a_{i}$. A cycle is simple if all variables, except the first and last one, are pairwise distinct. According to Shostak lemma [17], a system of inequalities $\varphi$ has a solution iff every cycle in $\varphi$ has non-negative weight iff every simple cycle in $\varphi$ has non-negative weight. Detection of cycles of negative weight can be efficiently performed with the Bellman-Ford algorithm.

Proof (of Prop. 6.). We will consider three systems of inequalities.

1. The first one is $\phi_{1}=\Phi(\rho \cdot n)$.

2. The second one is $\phi_{2}=\Phi_{K_{2}, K_{2}}(\rho \cdot n)$. By definition, $\phi_{2}$ is obtained from $\phi_{1}$ by adding inequalities $x_{e}-x_{f} \leq K_{2}$ for all $e, f$ from the same node of $\rho \cdot n$.

3. Finally, $\phi_{3}=\Phi_{K, \widehat{K}}(\rho)$. Since $K \leq \widehat{K} \leq K_{2}, \phi_{3}$ can be obtained from $\phi_{2}$ by deleting the events from $n$, and adding inequalities $x_{e}-x_{f} \leq K$ for all concrete $e, f$ from the same node of $\rho$, and adding inequalities $x_{e}-x_{f} \leq \widehat{K}$ for all events $e, f$ from the same node of $\rho$ s.t. $e$ or $f$ or both are dummy.

We know that $\rho \cdot n$ is consistent iff $\phi_{1}$ is satisfiable. Hence, we just need to prove that $\phi_{2}$ has a solution iff $\phi_{1}$ has a solution to yield the statement of the proposition. Clearly, if $\phi_{2}$ has a solution, then this solution is also a solution for $\phi_{1}$. Conversely, assume that $\phi_{1}$ has a solution. By Shostak lemma, it implies that every cycle in $\phi_{1}$ has weight at least 0 . Now to prove that $\phi_{2}$ has a solution, it suffices to show that every simple cycle of $\phi_{2}$ has weight at least 0 . Let $x_{1} \ldots x_{m}$ be a simple cycle in $\phi_{2}$. That is, for all $i \in\{1, \ldots, m-1\}, x_{i+1}-x_{i} \leq b_{i}$ appears in $\phi_{2}$ for some $b_{i}$, and $x_{m}=x_{1}$. We want to prove that $\sum_{i} b_{i} \geq 0$.

Let $a_{i}$ be the associated coefficients in $\phi_{1}$, i.e such that there is an inequality in $\phi_{1}$ of the form, $x_{i+1}-x_{i} \leq a_{i}$ (if $a_{i}$ does not exist, fix $a_{i}=+\infty$ ). Let $c_{i}$ be 
the associated coefficients of $\phi_{3}$ (we fix $c_{i}=-\infty$ if it corresponds to events in $n$, i.e., events not represented in $\left.\phi_{3}\right)$.

Observe that $c_{i} \leq b_{i} \leq a_{i}$ by definition of $\phi_{1}, \phi_{2}, \phi_{3}$. Now, if $a_{i}=b_{i}$ for all $i$, then the cycle $x_{1} \ldots x_{m}$ in $\phi_{2}$ is also a cycle in $\phi_{1}$ and $\sum_{i} b_{i}=\sum_{i} a_{i}$. As every cycle in $\phi_{1}$ has weight at least 0 , we are done. Else, we have $a_{j} \neq b_{j}$ for some $j$. Let $J \neq \emptyset$ be the set of indices $j$ such that $a_{j} \neq b_{j}$. Hence $|J| \geq 1$. Further, $e_{j}, e_{j+1}$ are in the same node $m$ of $\rho \cdot n$ for all $j \in J$, because $\phi_{2}$ only adds constraints on pairs of events of the same node. Last, $b_{j}=K_{2}$ for all $j \in J$, as the only additional constraints in $\phi_{2}$ w.r.t. $\phi_{1}$ are of the form $x_{e}-x_{f} \leq K_{2}$.

Now, we partition the indices $\{1, \ldots, m\}=I_{\rho} \cup I_{n} \cup J_{\rho} \cup J_{n}$ where, $J_{\rho}=\left\{j \mid b_{j}=K_{2}\right.$ and both $x_{j}$ and $x_{j+1}$ belong to $\left.\rho\right\}$,

$J_{n}=\left\{j \mid b_{j}=K_{2}\right.$ and at least one of $x_{j}$ or $x_{j+1}$ belongs to $\left.n\right\}$.

$I_{n}=\left\{j \mid b_{j} \neq K_{2}\right.$ and at least one of $x_{j}$ or $x_{j+1}$ belongs to $\left.n\right\}$, and $I_{\rho}=\left\{j \mid b_{j} \neq K_{2}\right.$ and both $x_{j}$ and $x_{j+1}$ belong to $\left.\rho\right\}$.

With this $\sum_{i} b_{i}=\sum_{i \in I_{n}} b_{i}+\sum_{i \in J_{n}} b_{i}+\sum_{i \in I_{\rho}} b_{i}+\sum_{i \in J_{\rho}} b_{i}$. Now, observing that $J=J_{\rho} \cup J_{n}$, we have $\sum_{i \in\left(J_{n} \cup J_{\rho}\right)} b_{i}=K_{2} \cdot\left(\left|J_{\rho}\right|+\left|J_{n}\right|\right)=K_{2} \cdot|J| \geq K_{2} \cdot 1$. Further, we also have $\sum_{i \in I_{n}} b_{i} \geq-D(\widehat{G})$ by definition of $D(\widehat{G})$ and because the cycle is simple. Now, we bound the sum $\sum_{i \in I_{\rho}} b_{i}$ (the remaining weights) using $\phi_{3}$. Indeed, since each $i \in I_{\rho}$ is an index such that $x_{i}$ and $x_{i+1}$ are events of $\rho$, we have $b_{i} \geq c_{i}$ where $c_{i}$ is the coefficient of $\phi_{3}$. And therefore it suffices to bound $\left(\sum_{i \in I_{\rho}} c_{i}\right)$. It immediately yields the bound for $\sum_{i} b_{i}$.

For this, the set $I_{\rho}$ is first partitioned into pieces. Each piece $I^{\prime} \subseteq I_{\rho}$ is made of "consecutive" indices, i.e., either $I^{\prime}=\{i, i+1 \ldots, j\}$ or $I^{\prime}=\{i, \ldots, m, 1, \ldots, j\}$, such that $\left(e_{i-1} \in n\right.$ or $\left.b_{i}=K_{2}\right)$ and $\left(e_{j+1} \in n\right.$ or $\left.b_{j}=K_{2}\right)$. There are at most $\left|J_{\rho}\right|+|\mathcal{P}| / 2$ pieces (because the cycle is simple). Each piece begins and ends either with the last event on some process of the node before $n$ or with an event $e_{i}$ or $e_{i+1}$ such that $b_{i}=K_{2}$.

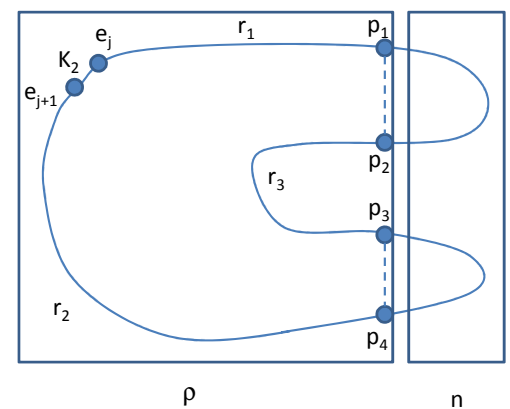

For instance, the picture above depicts a cycle (in $\left.\phi_{2}\right)$ with 3 pieces $r_{1}, r_{2}, r_{3}$, involving 4 processes. $r_{1}$ begins with the last event on some process $p_{1}$ of $\rho$ and ends with an event $e_{j}$ such that $b_{j}=K_{2} \cdot r_{2}$ begins with $e_{j+1}$ and ends with the last event on some process $p_{4}$ of $\rho . r_{3}$ begins and ends with the last events on some processes $p_{2}, p_{3}$ of $\rho$. 
As $\rho$ is $(K, \widehat{K})$-drift-bounded and consistent, we know that $\phi_{3}$ has a solution, that is every cycle in $\phi_{3}$ has weight at least 0 by Shostak lemma. Let $I_{1}, \cdots, I_{r}$ be the pieces of $I_{\rho}$. Recall that $r \leq\left|J_{\rho}\right|+|\mathcal{P}| / 2$. For all $i \leq r$, denoting $I_{i}=\{s, \ldots, t\}$, we rename $e_{s} \cdots e_{t}$ into $y_{1}^{i} \cdots y_{m^{i}}^{i}$. We now build a cycle of $\phi_{3}$ using every piece, and with some additional edges connecting these pieces. More precisely, we define $\xi=y_{1}^{1} \cdots y_{m^{1}}^{1} \cdots y_{1}^{r} \cdots y_{m^{r}}^{r} y_{1}^{1}$, made by gluing all the pieces together. Comparing the weight of $\xi$ with $\sum_{i \in I_{\rho}} c_{i}$, there is an additional weight $d_{i}$ in $\xi$ with $y_{1}^{i+1}-y_{m^{i}}^{i} \leq d_{i}$, for each $i$. We have that both $y_{m^{i}}^{i}$ and $y_{1}^{i+1}$ are in the same node (either the last node before $n$, or some node where there were a $K_{2}$ edge). In $\phi_{3}$, there is an edge between any two events of the same node, hence this connecting edge $y_{1}^{s+1}-y_{m^{s}}^{s} \leq c_{s}$ exists (that is $\xi$ is a cycle), and $c_{s} \leq \widehat{K}$, by definition of $\phi_{3}$. By Shostak lemma, the weight $w$ of $\xi$ in $\phi_{3}$ is at least 0 . We thus have $\left(\sum_{i \in I_{\rho}} c_{i}\right)+\left(|\mathcal{P}| / 2+\left|J_{\rho}\right|\right) \cdot \widehat{K} \geq w \geq 0$. We then have $\sum_{i \in I_{\rho}} b_{i} \geq \sum_{i \in I_{\rho}} c_{i} \geq-\left(|\mathcal{P}| / 2+\left|J_{p}\right|\right) \cdot \widehat{K}$. Thus, we get $\sum_{i} b_{i} \geq K_{2} \cdot\left(\left|J_{\rho}\right|+\left|J_{n}\right|\right)-$ $\left.\left(|\mathcal{P}| / 2+\left|J_{\rho}\right|\right) \cdot \widehat{K}-D(\widehat{G})=K_{2}+\left(\left|J_{\rho}\right|+\left|J_{n}\right|-1\right) \cdot K_{2}-(D(\widehat{G})+|\mathcal{P}| / 2 K)-\left|J_{\rho}\right|\right) \cdot \widehat{K}=$ $\widehat{K}+\left(\left|J_{\rho}\right|+\left|J_{n}\right|-1\right) \cdot K_{2}-\left|J_{\rho}\right| \cdot \widehat{K}=\left(\left|J_{\rho}\right|+\left|J_{n}\right|-1\right) \cdot K_{2}-\left(\left|J_{\rho}\right|-1\right) \cdot \widehat{K} \geq 0$, as $\left|J_{\rho}\right|-1 \leq\left|J_{\rho}\right|+\left|J_{n}\right|-1,0 \leq\left|J_{\rho}\right|+\left|J_{n}\right|-1$ and $\widehat{K} \leq K_{2}$.

We can now build an automaton accepting minimal witness paths of $\widehat{G}$.

An automaton for minimal witnesses. We search for a minimal witness path $\rho \cdot n=n_{0} \cdots n_{\ell} \cdot n$ in $\widehat{G}$ using an automaton $\mathcal{B}(\widehat{G})$. The first component of a state of $\mathcal{B}(\widehat{G})$ keeps track of the current node $n$. The second component will test for $(K, \widehat{K})$-drift-boundedness, which needs to hold for $\rho$ but not for $\rho \cdot n$. This is done by keeping track of a $\widehat{K}$-bounded profile. The last component keeps track of $P F_{K_{2}, K_{2}}(\rho)$ which is sufficient to check consistency of $\rho$ according to Proposition 6 . Theorem 2 is obtained using the following proposition (where $|\widehat{G}|$ is the number of nodes of $\widehat{G}$ ):

Proposition 7. Let $G$ be a $T C-M S C$ graph. Then there exists an automaton $\mathcal{B}(\widehat{G})$ such that $\mathcal{L}(\mathcal{B}(\widehat{G}))=\emptyset$ iff $\widehat{G}$ is $(K, \widehat{K})$-drift-bounded. Further, $\mathcal{B}(\widehat{G})$ has at most $|\widehat{G}| \times(2 \widehat{K}+1)^{|\mathcal{P}|^{2}} \times\left(2 K_{2}+1\right)^{|\mathcal{P}|^{2}}$ states, where $K_{2}=(|\mathcal{P}| / 2+1) \cdot \widehat{K}+D(\widehat{G})$.

Proof (Sketch). The states of $\mathcal{B}(\widehat{G})$ are triples $(n, \sigma, \tau)$, with $n$ a node of $\widehat{G}, \sigma$ a $\widehat{K}$-bounded profile of $\widehat{G}$, and $\tau$ a $K_{2}$-bounded profile of $\widehat{G}$. The initial state of $\mathcal{B}(\widehat{G})$ is $\left(n_{i n}, P F_{K, \widehat{K}}\left(n_{i n}\right), P F_{K_{2}, K_{2}}\left(n_{i n}\right)\right)$. A state $(n, \sigma, \tau)$ of $\mathcal{B}(\widehat{G})$ is final if $\sigma$ is not satisfiable, but $\tau$ is. Last, there is a transition labeled by $n^{\prime}$ from $(n, \sigma, \tau)$ to $\left(n^{\prime}, \sigma^{\prime}, \tau^{\prime}\right)$ iff $\widehat{G}$ contains a transition $n \rightarrow n^{\prime}, \sigma^{\prime}=\theta_{K, \widehat{K}}^{n \rightarrow n^{\prime}}(\sigma), \tau^{\prime}=\theta_{K_{2}, K_{2}}^{n \rightarrow n^{\prime}}(\tau)$, and both $\sigma$ and $\tau^{\prime}$ are satisfiable. Notice that $\tau$ is satisfiable when $\sigma$ is, as $K_{2} \geq \widehat{K} \geq K$, and that $\sigma^{\prime}$ is not required to be satisfiable.

\section{Conclusion}

This paper has addressed the emptiness problem for TC-MSC graphs. We have shown that emptiness can be checked under the restriction that a TC-MSC graph 
is $K$-drift-bounded, for some $K$, and we established the decidability of checking this restriction. The decision procedure does not consider linearizations of TCMSC graphs, nor rely on the seminal result of [4]. Instead, a finite automaton keeps track of a system of inequalities describing symbolically constraints over dates on each process. As future work, we plan to consider checking whether a TC-MSC graph is drift-bounded (without the bound $K$ ), and if so computing the bound. It seems that tackling this problem needs new ideas and concepts.

\section{References}

1. S. Akshay, B. Genest, L. Hélouët, and S. Yang. Regular set of representatives for time-constrained MSC graphs. Inf. Proc. Letters, 112(14-15):592-598, 2012.

2. S. Akshay, M. Mukund, and K. Narayan Kumar. Checking coverage for infinite collections of timed scenarios. In CONCUR 2007, LNCS 4703, pp. 181-196. Springer.

3. S. Akshay, P. Gastin, K. Narayan Kumar, and M. Mukund. Model checking time-constrained scenario-based specifications. In FSTTCS 2010, LNCS 4855, pp. 290-302. Springer.

4. R. Alur and D. L. Dill. A theory of timed automata. Theoretical Comp. Sci., 126(2):183-235, 1994.

5. R. Alur and M. Yannakakis. Model checking of message sequence charts. In CONCUR 1999, LNCS 1664, pp. 114-129. Springer.

6. J. Bengtsson and W. Yi. On clock difference constraints and termination in reachability analysis of timed automata. In ICFEM 2003, pp. 491-503.

7. P. Bouyer, S. Haddad, and P.-A. Reynier. Timed unfoldings for networks of timed automata. In ATVA 2006, LNCS 4218, pp. 292-306. Springer.

8. F. Cassez, T. Chatain, and C. Jard. Symbolic unfoldings for networks of timed automata. In ATVA 2006, LNCS 4218, pp. 307-321. Springer.

9. G. Dantzig and B. C. Eaves. Fourier-Motzkin Elimination and Its Dual. J. Comb. Theory, Ser. A, 14(3):288-297, 1973.

10. C. Dima and R. Lanotte. Distributed time-asynchronous automata. In ICTAC 2007, LNCS 4711, 185-200. Springer.

11. P. Gastin, K. Narayan Kumar, and M. Mukund. Reachability and boundedness in time-constrained MSC graphs. In Perspectives in Concurrency - A Festschrift for P. S. Thiagarajan. Universities Press, India, 2009.

12. J. G. Henriksen, M. Mukund, K. N. Kumar, M. Sohoni, and P. S. Thiagarajan. A theory of regular MSC languages. Inf. and Comp., 202(1):1-38, 2005.

13. ITU-TS Recommendation Z.120: Message Sequence Chart (MSC '99), 1999.

14. B. Korte and J. Vygen. Combinatorial Optimization: Theory and Algorithms. Springer, 3rd edition, 2006.

15. D. Lugiez, P. Niebert, and S. Zennou. A partial order semantics approach to the clock explosion problem of timed automata. Theoretical Comp. Sci., 345(1):27-59, 2005.

16. A. Muscholl and D. Peled. Message sequence graphs and decision problems on Mazurkiewicz traces. In MFCS 1999, LNCS 1672, pp. 81-91. Springer.

17. R. Shostak. Deciding linear inequalities by computing loop residues. JACM, 28(4):769-779, 1981.

18. J. Zhao, H. Xu, X. Li, T. Zheng, and G. Zheng. Partial order path technique for checking parallel timed automata. In FTRTFT 2002, LNCS 2469, pp. 417-432. 


\section{Appendix}

Additional material (mainly proofs) is given section-wise.

\subsection{Full TC-MSC Graphs}

In this subsection, we show the properties relating a TC-MSC graph $G$ and its "augmented" full TC-MSC graph $\widehat{G}$ obtained by adding "dummy events" to nodes of $G$. For notational convenience, we assume that TC-MSCs may contain internal events. Indeed, as is standard, an internal event can be simulated by a send event to some new process (not already in $\mathcal{P}$ ).

Proposition 2. For a TC-MSC graph $G$, a path $\rho$ of $G$ and $K \in \mathbb{N}$, (i) $\rho$ is consistent in $G$ iff $\widehat{\rho}$ is consistent in $\widehat{G}$, (ii) $\rho$ is $K$-drift-bounded in $G$ iff $\widehat{\rho}$ is $(K, \widehat{K})$-drift-bounded in $\widehat{G}$, with $\widehat{K}=(|\mathcal{P}|-1) \cdot K$.

Proof: The proof of $(i)$ is straightforward. Any consistent path $\rho=n_{0} \ldots n_{\ell}$ of $\widehat{G}$ is also a consistent path in $G$, since if $M$ is a consistent dated MSC for $\rho$ in $\widehat{G}$, one can obtain a consistent dated MSC $M^{\prime}$ for $\rho$ in $G$ by deleting dummy events. Conversely, taking a consistent path $\rho$ of $G$ and a dated MSC $M$ for it, one can create a consistent dated MSC $M^{\prime} \in \mathcal{L}(\widehat{G})$ for $\rho$ in $\widehat{G}$ from $M$ by adding the dummy events and setting the date of dummy event $e$ on $p$ to be the same as the date of the event on $p$ immediately before $e$ (or date 0 if there is no such event).

One direction of proof of $(i i)$ is also trivial, since if $\widehat{\rho}$ is $(K, \widehat{K})$-drift-bounded in $\widehat{G}$, then by deleting the dummy events, we obtain that $\rho$ is $K$-drift-bounded in $G$. The other direction of (ii) is much more involved, and will be infered from the three technical lemmas that follow.

Lemma 2. Let $\rho=n_{0} \ldots n_{\ell}$ be a $K$-drift-bounded path of $G$ such that for every $h=0, \ldots, \ell-1$, there exists some process $p_{h}$, such that both $n_{h}, n_{h+1}$ have events on $p_{h}$. Let $\left(E,\left(<_{p}\right), \mu, \lambda, d\right)$ be a $K$-drift-bounded dated MSC generated by $\rho$. Then for any indices $i, j$ with $0 \leq i<j \leq \ell$, if $e$ is an event in $n_{i}, f$ an event in $n_{j}$, then $d(e)-d(f) \leq \widehat{K}$.

Note that we do not claim $|d(e)-d(f)| \leq \widehat{K}$, which may not be true in general. Intuitively, Lemma 2 just means that, under the given hypothesis, an event $e$ appearing in a node $n_{i}$ cannot be associated a date which is too ahead of the dates of events appearing in the subsequent nodes in the path.

Proof. By the hypothesis of the lemma, one can choose a sequence of processes $p_{i} \ldots p_{j-1}$, such that for each $h=i, \ldots, j-1, n_{h}, n_{h+1}$ both have events on process $p_{h}$. From the sequence $p_{i} \ldots p_{j-1}$, we pick a subsequence $p_{\alpha_{1}} \ldots p_{\alpha_{z}}$, where $z \leq|\mathcal{P}|$, as follows. Firstly, let $\alpha_{1}$ be the largest index in $\{i, \ldots, j-1\}$, such that $p_{\alpha_{1}}=p_{i}$. That is, $p_{h} \neq p_{i}$ whenever $\alpha_{1}<h \leq j-1$. Secondly, inductively, for $u=1, \ldots$, suppose $\alpha_{1}, \ldots, \alpha_{u}$ have been set. Pick $\alpha_{u+1}$ to be the largest index in $\left\{\alpha_{u}+1, \ldots, j-1\right\}$ such that $p_{\left(\alpha_{(u+1)}\right)}=p_{\left(\left(\alpha_{u}\right)+1\right)}$. That is, $p_{h} \neq p_{\alpha_{u}+1}$ whenever 
$\alpha_{u+1}<h \leq j-1$. It follows that $p_{\alpha_{1}}, p_{\alpha_{2}}, \ldots$, are pairwise distinct, and thus this procedure of picking indices $\alpha_{1}, \alpha_{2}, \ldots$ will terminate after picking $\alpha_{z}=j-1$ for some $z \leq|\mathcal{P}|$. We emphasize that $p_{\left(\alpha_{(u+1)}\right)}=p_{\left(\left(\alpha_{u}\right)+1\right)}$ for $u=1, \ldots, z-1$.

Now for $h=1, \ldots, z-1$, and a sequence $p_{i}, \ldots, p_{j-1}$ pick events $x_{h}, y_{h}$ from node $n_{\alpha_{h}+1}$ such that $x_{h}$ is on process $p_{\alpha_{h}}$ and $y_{h}$ is on process $p_{\alpha_{h}+1}$. Further, pick event $y_{0}$ on process $p_{i}$ from $n_{i}$ and event $x_{z}$ on process $p_{j-1}$ from $n_{j}$. Existence of $x_{h}, y_{h}, h=1, \ldots, z-1$, and $y_{0}, x_{z}$ is guaranteed by construction of the sequence $p_{i}, \ldots, p_{j}$. Set $x_{0}=e$ and $y_{z}=f$. For $h=0, \ldots, z-1$, since $y_{h}$, $x_{h+1}$ are of the same process, we have $d\left(y_{h}\right) \leq d\left(x_{h+1}\right)$. Since $\left(E,\left(<_{p}\right), \mu, \lambda, d\right)$ is $K$-drift-bounded, we have $d\left(x_{h}\right)-d\left(y_{h}\right) \leq K$ for $h=0, \ldots, z$. Suppose $e$ is on process $p_{e}$ and $f$ on process $p_{f}$. Recall that $p_{\alpha_{1}}, \ldots, p_{\alpha_{z}}$ are pairwise distinct. We show $d(e)-d(f) \leq \widehat{K}$ by considering four cases.

- Case (1). If $p_{e}, p_{\alpha_{1}}, \ldots, p_{\alpha_{z}}, p_{f}$ are pairwise distinct, then $z \leq|\mathcal{P}|-2$, and thus $d(e)-d(f) \leq \sum_{h=0}^{z}\left(d\left(x_{h}\right)-d\left(y_{h}\right)+\sum_{h=0}^{z-1}\left(d\left(y_{h}\right)-d\left(x_{h+1}\right)\right) \leq(z+1) \cdot K \leq \widehat{K}\right.$.

- Case (2). If $p_{e}=p_{\alpha_{t}}$ for some $t$ in $\{1, \ldots, z\}$ and $p_{\alpha_{1}}, \ldots, p_{\alpha_{z}}, p_{f}$ are pairwise distinct, then $z \leq|\mathcal{P}|-1$ and thus $d(e)-d(f) \leq d(e)-d\left(x_{t}\right)+$ $\sum_{h=t}^{z} d\left(x_{h}\right)-d\left(y_{h}\right) \leq(z-t+1) \cdot K \leq \widehat{K}$.

-There remains two cases: (i) $p_{e}, p_{\alpha_{1}}, \ldots, p_{\alpha_{z}}$ are distinct, $p_{f}=p_{\alpha_{t}}$ for some $t$ in $\{1, \ldots, z\}$. (ii) $p_{e}=p_{\alpha_{t}}, p_{f}=p_{\alpha_{u}}$ for some $t, u \in\{1, \ldots, z\}$. Both cases can be handled similarly to cases (1) and (2), which completes the proof of Lemma 2.

The above lemma motivates a new notion, which will turn out to be crucial in what follows. Let $\rho=n_{0} \ldots n_{\ell}$ be a path of $G$, and $\left(E,\left(<_{p}\right), \mu, \lambda, d\right)$ a dated MSC generated by $\rho$. For an integer $C$, we define $\left(E,\left(<_{p}\right), \mu, \lambda, d\right)$ to be $C$-distant iff for any $i, j$ in $\{0, \ldots, \ell\}$ with $i<j$, for any event $e$ in $n_{i}, f$ in $n_{j}$, it is the case that $d(e)-d(f) \leq C$. Note that unlike $K$-drift-boundedness, the notion of being $C$-distant places restriction on dates of events in two different nodes. Intuitively, being $C$-distant means if event $e$ is at node which occurs earlier than the node in which event $f$ is in, then $e$ can be executed at most $C$ time units later than $f$.

Lemma 3. Suppose that $\rho$ is a $K$-drift-bounded consistent path of $G$. Then there exists a $\widehat{K}$-distant $K$-drift-bounded dated $M S C$ generated by $\rho$ in $G$.

Proof. Let $\left(E,\left(<_{p}\right), \mu, \lambda, d\right)$ be a $K$-drift-bounded dated MSC generated by $\rho=n_{0} \ldots n_{\ell}$. If for every $h=0, \ldots, \ell-1, n_{h}$ and $n_{h+1}$ have events on some process $p_{h}$, then by Lemma $2,\left(E,\left(<_{p}\right), \mu, \lambda, d\right)$ is $\widehat{K}$-distant. Now suppose such is not the case. Let $t_{1}<\ldots<t_{z}$ be all the indices in $\{0, \ldots, \ell-1\}$ such that the set of events of $n_{t_{i}}$ and $n_{t_{i}+1}$ occur on a disjoint set of processes. From the proof of Lemma 2 it follows that, if $e$ is an event in $n_{i}, f$ an event in $n_{j}$, and none of $t_{1}, \ldots, t_{z}$ falls within $\{i, \ldots, j-1\}$, then $d(e)-d(f) \leq \widehat{K}$.

Observe that for each $i=1, \ldots, z$, there is no time constraint dictated between an event in $n_{0}, \ldots, n_{t_{i}}$ and an event in $n_{t_{i}+1}, \ldots, n_{\ell}$. Fix an integer $c$ whose choice is to be determined later. From $\left(E,\left(<_{p}\right), \mu, \lambda, d\right)$, we construct a new dated MSC $\left(E,\left(<_{p}\right), \mu, \lambda, d^{\prime}\right)$ by inductively applying the modifications associated with $t_{1}, \ldots, t_{z}$ as follows. Firstly, we apply the modification associated with $t_{1}$, which is to add $c$ to the date of each event in $n_{t_{1}+1}, \ldots, n_{\ell}$ (while the date of 
any event in $n_{0}, \ldots, n_{t_{1}}$ remains unchanged). Inductively, suppose modifications associated with $t_{1}, \ldots, t_{i-1}$ have been done, for some $i \leq z$. We further apply the modification associated with $t_{i}$, which is to add $c$ to the date of each event in $n_{t_{i}+1}, \ldots, n_{\ell}$ (while the date of any event in $n_{0}, \ldots, n_{t_{i}}$ remains unchanged).

Note that the date of an event is non-negative. By choosing $c$ such that $d(g)-\widehat{K} \leq c$ for every event $g$ in $n_{0} \ldots, n_{\ell}$, one concludes that in $\left(E,\left(<_{p}\right), \mu, \lambda, d^{\prime}\right)$, for any event $e$ in $n_{i}, f$ in $n_{j}$, with $i<j$, and some of the indices $t_{1}, \ldots, t_{z}$ falling within $\{i, \ldots, j-1\}$, we have $d^{\prime}(e)-d^{\prime}(f) \leq d(e)-c \leq \widehat{K}$. If none of the indices $t_{1}, \ldots, t_{z}$ falls within $\{i, \ldots, j-1\}$, then $d^{\prime}(e)-d^{\prime}(f)=d(e)-d(f) \leq \widehat{K}$ as observed earlier, following the proof of lemma 2. Clearly, $\left(E,\left(<_{p}\right), \mu, \lambda, d^{\prime}\right)$ is $K$-drift-bounded and fulfills the time constraints in $\rho$, since $\left(E,\left(<_{p}\right), \mu, \lambda, d\right)$ is a $K$-drift-bounded dated MSC generated by $\rho$. This completes the proof.

The next lemma shows that $K$-drift-bounded and $\widehat{K}$-distant dated MSCs of $G$ can be transformed into $(K, \widehat{K})$-drift-bounded dated MSCs of $\widehat{G}$. Together with Lemma 3, one establishes that if $\rho$ is a $K$-drift-bounded path of $G$, then $\widehat{\rho}$ is a $(K, \widehat{K})$-drift-bounded path of $\widehat{G}$, thus completing the proof of the remaining part of Proposition 2(ii).

Lemma 4. Assume that there exists a dated MSC generated by a consistent path $\rho$ of $G$, which is $\widehat{K}$-distant and $K$-drift-bounded. Then one can construct $a(K, \widehat{K})$-drift-bounded dated $M S C$ of $\widehat{G}$, which is in $\mathcal{L}\left(T^{\widehat{\rho}}\right)$ where $T^{\widehat{\rho}}$ is the TC-MSC generated by $\widehat{\rho}$ in $\widehat{G}$.

Proof. Let $\rho=n_{0} \ldots n_{\ell}$, and let $M=\left(E,\left(<_{p}\right), \mu, \lambda, d\right)$ be a $\widehat{K}$-distant $K$-driftbounded dated MSC generated by $\rho$ in $G$. Recall the construction of $\widehat{G}=$ $\left(N, \widehat{\mathcal{T}}, \widehat{\Lambda}, n_{i n}, N_{f i}, \longrightarrow, \Delta\right)$ from the beginning of Section 3.2. We shall extend $M$ to be to a dated MSC $M^{\prime}=\left(E^{\prime},\left(<_{p}\right), \mu, \lambda, d^{\prime}\right)$ generated by $\widehat{\rho}$ in $\widehat{G}$ as follows. First, $E^{\prime}$ consists of events in $T^{\widehat{\rho}}$, the TC-MSC obtained by concatenation of nodes of $\rho$ according to $\widehat{G}$. Second, we keep dates of events in $E$ unchanged (that is, $d^{\prime}(e)=d(e)$ for every $\left.e \in E\right)$, and assign suitable dates to dummy events. The assignment of dates to dummy events are done inductively, node by node, for nodes $n_{0}, \ldots, n_{\ell}$. Through the rest of this proof, for each $i=0, \ldots, \ell$, pick an event $f_{i}^{\max }$ in $n_{i}$ which has maximum date among events in $n_{i}$. For node $n_{0}$, for any dummy event $e$ in $\widehat{\Lambda}\left(n_{0}\right)$, we set $d^{\prime}(e)=\max \left\{d\left(f_{0}^{\max }\right)-\widehat{K}, 0\right\}$. Inductively, assume that dummy events in $\widehat{\Lambda}\left(n_{0}\right), \ldots, \widehat{\Lambda}\left(n_{i-1}\right)$ have been assigned dates, then for any dummy event $e$ in $\widehat{\Lambda}\left(n_{i}\right)$, we set $d^{\prime}(e)$ to be the larger of $d^{\prime}\left(e_{i-1}\right)$ and $d\left(f_{i}^{\text {max }}\right)-\widehat{K}$, where $e_{i-1}$ is the maximal event in $\widehat{\Lambda}\left(n_{i-1}\right)$ which is on the same process as $e$. Note that $e_{i-1}$ exists as $\widehat{\Lambda}\left(n_{i-1}\right)$ is full.

Since concrete events in $M^{\prime}$ has the same dates as in $M$, to see that $M^{\prime}$ satisfies the time constraints in $\widehat{\rho}$, it suffices to show:

Claim (1): For any $i=0, \ldots, \ell-1$, for any process $p$, if at least one of $\widehat{\Lambda}\left(n_{i}\right), \widehat{\Lambda}\left(n_{i+1}\right)$ contains a dummy event on $p$, then $d^{\prime}\left(e_{i}\right) \leq d^{\prime}\left(e_{i+1}\right)$ where $e_{i}$ is the maximal event on $p$ in $\widehat{\Lambda}\left(n_{i}\right)$, and $e_{i+1}$ the minimal event on $p$ in $\widehat{\Lambda}\left(n_{i+1}\right)$.

We now prove Claim (1). Fix $i, p$. If $e_{i+1}$ is a dummy event, then by definition of $d^{\prime}\left(e_{i+1}\right)$, we have $d^{\prime}\left(e_{i}\right) \leq d^{\prime}\left(e_{i+1}\right)$. It remains to consider the case that $e_{i}$ is a 
dummy event but $e_{i+1}$ is not a dummy event. Let $j$ be the largest index such that $0 \leq j<i$ and $\widehat{\Lambda}\left(n_{j}\right)$ contains concrete events on $p$. If such a $j$ exists, set $D=d\left(e_{j}\right)$ where $e_{j}$ is the maximal event on $p$ in $\widehat{\Lambda}\left(n_{j}\right)$ (which is an concrete event); if no such $j$ exists, set $j=-1$ and $D=0$. By "unrolling" the definition of $d^{\prime}\left(e_{i}\right)$, one sees that $d^{\prime}\left(e_{i}\right)$ is the maximum in the set consisting of $D$ and $d\left(f_{h}^{\max }\right)-\widehat{K}$ for all indices $h$ with $j<h \leq i$. Since $e_{i+1}$ is on $p$, the choice of $D$ ensures that $D \leq d\left(e_{i+1}\right)$. Owing to that $\rho$ is $\widehat{K}$-distant, we have $d\left(f_{h}^{\max }\right)-\widehat{K} \leq d\left(e_{i+1}\right)$ whenever $j<h \leq i$. These yield that $d^{\prime}\left(e_{i}\right) \leq d\left(e_{i+1}\right)=d^{\prime}\left(e_{i+1}\right)$. - End of proof of Claim (1)

Having shown that $M^{\prime}$ is a dated MSC generated by $\widehat{\rho}$ in $\widehat{G}$, we next prove that $M^{\prime}$ is $(K, \widehat{K})$-drift-bounded. Since $M$ is $K$-drift-bounded and the dates of concrete (non-dummy) events in $M, M^{\prime}$ are the same, it suffices to show:

Claim (2): For nodes $n_{0}, \ldots, n_{\ell}$ in $\rho$, if $e, g$ are events in $\widehat{\Lambda}\left(n_{i}\right)$ such that at least one of $e, g$ is a dummy event, then $\left|d^{\prime}(e)-d^{\prime}(g)\right| \leq \widehat{K}$.

We prove Claim (2) by induction on $i$. For $i=0$, let $e, g$ be events in $\widehat{\Lambda}\left(n_{0}\right)$ such that at least one of them is a dummy event. Suppose $e$ is dummy. If $g$ is also dummy, then $d^{\prime}(g)=d^{\prime}(e)$, else $d^{\prime}(e)=\max \left\{d\left(f_{0}^{\max }\right)-\widehat{K}, 0\right\}, d^{\prime}(g)=d(g)$ and $d\left(f_{0}^{\max }\right)-K \leq d(g) \leq d\left(f_{0}^{\max }\right)$ would imply that $\left|d^{\prime}(e)-d^{\prime}(g)\right| \leq \widehat{K}$.

Assume now that Claim (2) holds for node $n_{0}, \ldots, n_{i-1}$. Let $e, g$ be events in $\widehat{\Lambda}\left(n_{i}\right)$ such that at least one of them is dummy. Suppose $e$ is dummy. Let $e_{i-1}$ (resp. $g_{i-1}$ ) be the maximal event in $\widehat{\Lambda}\left(n_{i-1}\right)$ on the same process as $e$ (resp. $g$ ).

- Case (1): $g$ is not a dummy event.

If $d^{\prime}(e)=d\left(f_{i}^{\max }\right)-\widehat{K}$, then the same argument as in the base case of node $n_{0}$ yields that $\left|d^{\prime}(e)-d^{\prime}(g)\right| \leq \widehat{K}$. Otherwise, we have $d^{\prime}(e)=d^{\prime}\left(e_{i-1}\right)$. We have $d^{\prime}(e)-d^{\prime}(g) \leq d^{\prime}\left(e_{i-1}\right)-d^{\prime}\left(g_{i-1}\right) \leq \widehat{K}$ by induction hypothesis. And $d^{\prime}(e)-d^{\prime}(g) \geq\left(d\left(f_{i}^{\max }\right)-\widehat{K}\right)-d\left(f_{i}^{\max }\right)=-\widehat{K}$. These yield $\left|d^{\prime}(e)-d^{\prime}(g)\right| \leq \widehat{K}$.

- Case (2): $g$ is a dummy event.

If $d^{\prime}(e)=d^{\prime}\left(e_{i-1}\right)$ and $d^{\prime}(g)=d^{\prime}\left(g_{i-1}\right)$, then by induction hypothesis, we have $\left|d^{\prime}(e)-d^{\prime}(g)\right| \leq \widehat{K}$. The case of $d^{\prime}(e)=d\left(f_{i}^{\max }\right)-\widehat{K}$ and $d^{\prime}(g)=d\left(f_{i}^{\max }\right)-\widehat{K}$ is trivial. So it remains to consider the case that exactly one of $d^{\prime}(e)=d^{\prime}\left(e_{i-1}\right)$, $d^{\prime}(g)=d^{\prime}\left(g_{i-1}\right)$ holds. Since both $e, g$ are dummy events, w.l.o.g. assume $d^{\prime}(e)=$ $d^{\prime}\left(e_{i-1}\right)$ but $d^{\prime}(g) \neq d^{\prime}\left(g_{i-1}\right)$. That is, $d^{\prime}(g)=d\left(f_{i}^{\max }\right)-\widehat{K}>d^{\prime}\left(g_{i-1}\right)$. Thus, $d^{\prime}(e)-d^{\prime}(g)<d^{\prime}\left(e_{i-1}\right)-d^{\prime}\left(g_{i-1}\right) \leq \widehat{K}$ by induction hypothesis, and $d^{\prime}(e)-d^{\prime}(g) \geq$ 0 by definition of $d^{\prime}(e)$. These yield $\left|d^{\prime}(e)-d^{\prime}(g)\right|<\widehat{K}$.

- End of proof of Claim (2)

From Claims (1),(2), and the fact that $M$ is $K$-drift-bounded, one concludes that $M^{\prime}$ is a $(K, \widehat{K})$-drift-bounded dated MSC generated by $\widehat{\rho}$. This completes the proof of Lemma 4 and thus finally (using Lemma 3 and the arguments above), completes the proof of Proposition 2.

Corollary 1. Given a $T C$-MSC graph $G$, (i) $\mathcal{L}(G) \neq \emptyset$ iff $\mathcal{L}(\widehat{G}) \neq \emptyset$, and (ii) $G$ is $K$-drift-bounded iff $\widehat{G}$ is $(K, \widehat{K})$-drift-bounded, where $\widehat{K}=(|P|-1) K$. 
Proof: For part $(i), \mathcal{L}(G) \neq \emptyset$ means that there exists a path $\rho$ in $G$ that is consistent. From Proposition 2, $\rho$ is also a consistent path of $\widehat{G}$, and hence $\mathcal{L}(\widehat{G}) \neq \emptyset$. Suppose that $\mathcal{L}(G)=\emptyset$, then it means that for every path $\rho$, one cannot find a consistent date for events in the TC-MSC $T^{\rho}$ generated from $\rho$ in $G$. And so, we cannot find a consistent date for events in the TC-MSC $T^{\prime \rho}$ generated from $\rho$ in $\widehat{G}$. Proving (ii) is also straightforward. If $G$ is $K$-drift-bounded, then every path $\rho$ of $G$ is $K$-drift-bounded, and by Proposition $2, \rho$ is $(K, \widehat{K})$-driftbounded in $\widehat{G}$. Conversely, if $\widehat{G}$ is $(K, \widehat{K})$-drift-bounded, then every path $\rho$ of $\widehat{G}$ is $(K, \widehat{K})$-drift-bounded, and by Proposition 2, $\rho$ is $K$-drift-bounded in $G$.

\section{Emptiness for $K$-Drift-Bounded TC-MSC Graphs}

Fourier-Motzkin elimination technique: We now describe this technique whose details may be found in $[9,14]$. Let $\phi=\left\{x_{i}-x_{j} \leq a_{i j}\right\}$ be a system of inequalities over a set of variabes $X$, and let $x_{k} \in X$ be a variable to eliminate from $\phi$. That is, we want to obtain a new system of inequalities $\phi^{\prime}$ over variables $X \backslash\left\{x_{k}\right\}$ that is equivalent with $\phi$. For this, we first partition $\phi$ into three distinct systems of inequalities $\phi=\phi_{1} \wedge \phi_{2} \wedge \phi_{3}$, where $\phi_{1}$ is the system of inequalities that do not involve $x_{k}, \phi_{2}$ is the system of inequalities $\wedge_{i \in I} x_{k}-x_{i} \leq a_{k i}$ that involve $x_{k}$ as first operand, and $\phi_{3}$ is the system of inequalities $\wedge_{j \in J} x_{j}-x_{k} \leq a_{j k}$ that involve $x_{k}$ as second operand. Then $\exists x_{k}, \phi_{2} \wedge \phi_{3}$ is equivalent to $\exists x_{k}, \min _{j \in J}\left(\left(x_{j}-a_{j k}\right)\right) \leq$ $x_{k} \leq \max _{i \in I}\left(\left(a_{k i}+x_{i}\right)\right)$. We can thus eliminate variable $x_{k}$ to obtain an equivalent formula $\min _{j \in J}\left(\left(x_{j}-a_{j k}\right)\right) \leq \max _{i \in I}\left(\left(a_{k i}+x_{i}\right)\right)$. This is equivalent to (the system of $|I| \times|J|$ inequalities defined by) $\psi=\wedge_{i \in I, j \in J}\left(x_{j}-x_{i}\right) \leq\left(a_{j k}+a_{k i}\right)$. Note that if both $a_{j k}, a_{k i}$ are integers, then so is $a_{j k}+a_{k i}$.

Note that this elimination is not just a simple projection on $X \backslash\left\{x_{k}\right\}$. It propagates constraints attached to $x_{k}$ on remaining variables and this is why the set of solutions (over $X \backslash\left\{x_{k}\right\}$ ) remains the same. Notice also that the number of inequalities of $\phi^{\prime}$ is at most $(|X|-1)^{2}$, after simplification of $\phi^{\prime}$ (i.e., replacing each $x-y \leq a \wedge x-y \leq a^{\prime}$ by $\left.x-y \leq \min \left(a, a^{\prime}\right)\right)$.

We can extend elimination to sets of variables. Let $\phi$ be a system of difference inequalities over $X \cup Y$. Let $\psi_{1}$ and $\psi_{2}$ be two systems of inequalities over $Y$ obtained from $\phi$ by repeatedly applying Fourier-Motzkin elimination of each variable in $X$, where the order in which variables of $X$ are eliminated is different. Then we may have $\psi_{1} \neq \psi_{2}$. However, $\operatorname{Sol}\left(\psi_{1}\right)=\operatorname{Sol}\left(\psi_{2}\right)$, denoting by $\operatorname{Sol}(\psi)$ the set of solutions of any system of inequalities $\psi$. This allows us to fix an order

when eliminating variables. For $F \subseteq X$, let $\phi_{\mid F}$ denote the (unique) system of inequalities over variables $F$ obtained by performing Fourier-Motzkin elimination of variables in $X \backslash F$ following the fixed order. Regardless of the order, $\phi$ and $\phi_{\mid F}$ are equivalent. Also, if $\phi$ has integral coefficients, then so does $\phi_{\mid F}$.

\section{Symbolic and Bounded Profiles.}

Proposition 3. PF $(\rho)$ is satisfiable iff $\rho$ is consistent. 
Proof. The proof follows easily from the properties of Fourier-Motzkin elimination. A profile is obtained by successive elimination of all variables except those representing dates of last events executed on each process. From a system of inequalities $\phi$, one hence obtains an equivalent system $\phi^{\prime}$ by eliminating a sequence of variables. So, $P F(\rho)$ is satisfiable iff $\Phi(\rho)$ is satisfiable and by definition of $\Phi(\rho)$, it is satisfiable iff $\rho$ is consistent, which completes the proof.

Proposition 4. Let $\rho$ be a path of a full TC-MSC graph $H$ and $Y, Y^{\prime}$ be two positive integers such that $Y \leq Y^{\prime}$. Then, (1) $P F_{Y, Y^{\prime}}(\rho) \in \mathcal{P} \mathcal{F}_{Y^{\prime}}$ and (2) $\rho$ is consistent and $\left(Y, Y^{\prime}\right)$-drift-bounded iff $P F_{Y, Y^{\prime}}(\rho)$ is satisfiable.

Proof. The first part follows directly from the definitions. For the second part, the proof follows on the same lines as Proposition 3 above. For a path $\rho$ one can compute a system of inequalities $\Phi_{Y, Y^{\prime}}(\rho)$ such that $\rho$ is $\left(Y, Y^{\prime}\right)$-drift-bounded iff $\Phi_{Y, Y^{\prime}}(\rho)$ is satisfiable. As for unbounded profiles, $P F_{Y, Y^{\prime}}(\rho)$ is obtained by successive applications of Fourier-Motzkin eliminations. Such variable eliminations preserve satisfiability of systems of inequations, hence $P F_{Y, Y^{\prime}}(\rho)$ is satisfiable iff $\Phi_{Y, Y^{\prime}}(\rho)$ is satisfiable iff $\rho$ is $\left(Y, Y^{\prime}\right)$-drift-bounded and consistent.

Lemma 1. Let $H$ be a full TC-MSC graph and $Y \leq Y^{\prime}$ be two integers. Then, for a path $\rho$ and a transition $n^{-} \rightarrow n$ where $n^{-}$is the last node of $\rho$, we have $P F_{Y, Y^{\prime}}(\rho \cdot n)$ and $\theta_{Y, Y^{\prime}}^{n^{-}}{ }^{n}\left(P F_{Y, Y^{\prime}}(\rho)\right)$ have the same set of solutions.

Proof. This proof essentially follows from the fact that the Fourier-Motzkin elimination is confluent with respect to solutions. That is, the order in which the variables are eliminated does not matter for the set of solutions, as long as the resultant set of equations are over the same set of variables.

Consider the system of inequalities $\Phi_{Y, Y^{\prime}}(\rho \cdot n)$ on variables $X_{E}$ associated with path $\rho \cdot n$. Let $E^{n}$ denote the set of events of the TC-MSC $T^{n}$. Also denote by $E^{\rho}$ the set of events of $T^{\rho}$ and let $E_{\text {last }}^{\rho}=\left\{e_{p} \mid p \in \mathcal{P}\right\}$ with $e_{p}$ the last event of $T^{\rho}$ on $p$. Finally, let $X^{\rho}, X^{n}, X_{\text {last }}^{\rho}$ be the variables associated respectively with sets of events $E^{\rho}, E^{n}, E_{\text {last }}^{\rho}$.

We can partition $\Phi_{Y, Y^{\prime}}(\rho \cdot n)=\phi_{1} \wedge \phi_{2}$ with, for each $i \in\{1,2\}$,

$\phi_{i}=\left(\bigwedge_{x_{e}, x_{f} \in R_{i} \cap A} x_{e}-x_{f} \leq \min \left\{a_{e, f}, Y\right\}\right) \wedge\left(\bigwedge_{x_{e}, x_{f} \in R_{i} \cap B} x_{e}-x_{f} \leq \min \left\{a_{e, f}, Y^{\prime}\right\}\right)$

- where $R_{1}=X^{\rho} \times X^{\rho}$ and $R_{2}=\left(X^{n} \times\left(X^{n} \cup X_{\text {last }}^{\rho}\right)\right) \cup\left(\left(X^{n} \cup X_{\text {last }}^{\rho}\right) \times X^{n}\right)$, - $A=\left\{\left(x_{e}, x_{f}\right) \mid e\right.$ and $f$ are concrete $\}$ and $B=\left\{\left(x_{e}, x_{f}\right) \mid e\right.$ or $f$ is dummy $\}$

Thus $\phi_{1}$ corresponds to those inequalities that are fully in $\rho$ while $\phi_{2}$ has the rest. Now, consider $\left.\Phi_{Y, Y^{\prime}}(\rho \cdot n)\right|_{X^{n} \cup X_{\text {last }}^{\rho}}$ where all variables from $X^{\rho} \backslash X_{\text {last }}^{\rho}$ have been eliminated. This elimination keeps inequalities in $\phi_{2}$ intact and so we have $\left.\Phi_{Y, Y^{\prime}}(\rho \cdot n)\right|_{X^{n} \cup X_{\text {last }}^{\rho}}=\left.\left(\phi_{1} \wedge \phi_{2}\right)\right|_{X^{n} \cup X_{\text {last }}^{\rho}}=\left.\phi_{1}\right|_{X_{\text {last }}^{\rho}} \wedge \phi_{2}$.

Now (1) $\phi_{2}=\psi_{n} \wedge \psi_{n^{-} \rightarrow n}$ and (2) $\left.\phi_{1}\right|_{X_{\text {last }}^{\rho}}$ precisely correspond to $P F(\rho)$, i.e., $\left.\phi_{1}\right|_{X_{\text {last }}^{\rho}}=\psi_{P F(\rho)}$, where $\psi_{n}, \psi_{n^{-} \rightarrow n}, \psi_{P F(\rho)}$ are from the definition of 
$\theta_{Y, Y^{\prime}}^{n^{-}}{ }^{n}\left(P F_{Y, Y^{\prime}}(\rho)\right)$. Thus, denoting by $X_{\text {last }}^{\rho \cdot n}$ the set of variables attached to last events in $E^{\rho} \cup E^{n}$, we can eliminate all variables from $X^{n} \cup X_{\text {last }}^{\rho} \backslash X_{\text {last }}^{\rho \cdot n}$, to get that the set of solutions of $P F(\rho \cdot n)$ and $\theta^{n^{-} \rightarrow n}(P F(\rho))$ coincide (syntactically, the profiles may be different as the elimination orders may be different).

\section{Construction of a Symbolic Automaton.}

Proposition 5. Let $H$ be a full TC-MSC graph. Then, for any positive integers $Y \leq Y^{\prime}$, there exists an automaton $\mathcal{A}(H)$ with at most $|H| \times\left(2 \cdot Y^{\prime}+1\right)^{|\mathcal{P}|^{2}}$ states, such that $\mathcal{L}(\mathcal{A}(H)) \neq \emptyset$ iff $H$ has a (consistent) final $\left(Y, Y^{\prime}\right)$-drift-bounded path.

Proof. Note that we define drift-boundedness only for a consistent path and so the reuse of the term consistent in the statement above is purely for emphasis.

- The states of $\mathcal{A}(H)$ are pairs $(n, \sigma)$, with $n$ a state of $H$ and $\sigma \in \mathcal{P F}_{Y^{\prime}}$.

- The initial state is $\left(n_{i n}, P F_{Y, Y^{\prime}}\left(n_{i n}\right)\right)$.

- A state $(n, \sigma)$ is final if $n$ is final, and $\sigma$ is satisfiable.

- There is a transition from $(n, \sigma)$ to $\left(n^{\prime}, \sigma^{\prime}\right)$ labeled by $n^{\prime}$ iff

- both $\sigma, \sigma^{\prime}$ are satisfiable, and

- there is a transition from $n$ to $n^{\prime}$, and

- $\sigma^{\prime}=\theta_{Y, Y^{\prime}}^{n \rightarrow n^{\prime}}(\sigma)$.

There are at most $\left(2 \cdot Y^{\prime}+1\right)^{\left|P^{2}\right|}$ such profiles, and $\mathcal{A}(H)$ has at most $|H| \times(2$. $\left.Y^{\prime}+1\right)^{|\mathcal{P}|^{2}}$ states. We claim that $\mathcal{A}(H)$ accepts exactly the set of final $\left(Y, Y^{\prime}\right)$ drift-bounded (and hence consistent) paths of $H$. (More precisely, we mean that $\rho$ is accepted by $\mathcal{A}(H)$ iff $n_{\text {in }} \cdot \rho$ is a final $\left(Y, Y^{\prime}\right)$-drift-bounded path of $H$, but we abuse notation slightly and replace $n_{i n} \cdot \rho$ by $\rho$ itself.)

To see the claim, first consider any $\left(Y, Y^{\prime}\right)$-drift-bounded and hence consistent path $\rho=n_{0} \cdots n_{\ell}$ of $H$. It is easy to see that at most one sequence of states $\left(n_{0}, \sigma_{0}\right) \cdots\left(n_{\ell}, \sigma_{\ell}\right)$ in $\mathcal{A}(H)$ can exist. We show now that such a sequence exists as the path is $\left(Y, Y^{\prime}\right)$-drift-bounded (and consistent). Indeed, by contradiction, if such sequence does not exist, then there exists an index $i<\ell$ such that $\left(n_{0}, \sigma_{0}\right) \cdots\left(n_{i}, \sigma_{i}\right)$ is a sequence of states of $\mathcal{A}(G)$, but $\theta_{Y, Y^{\prime}}^{n_{i} \rightarrow n_{i+1}}\left(\sigma_{i}\right)$ is not a valid profile. As $\rho$ is $\left(Y, Y^{\prime}\right)$-drift-bounded, we have that $\rho^{\prime}=n_{0} \cdots n_{i} \cdot n_{i+1}$ is also $\left(Y, Y^{\prime}\right)$-drift-bounded. Hence $\Phi_{Y, Y^{\prime}}\left(\rho^{\prime}\right)$ and $P F_{Y, Y^{\prime}}\left(\rho^{\prime}\right)$ are satisfiable. We have that $P F_{Y, Y^{\prime}}\left(\rho^{\prime}\right)$ is satisfiable, and thus $\theta_{Y, Y^{\prime}}^{n_{i} \rightarrow n_{i+1}}\left(\sigma_{i}\right)$ is a valid profile by Lemma 1 , which is a contradiction with what we stated above.

Now, assume that the consistent and $\left(Y, Y^{\prime}\right)$-drift-bounded path $\rho$ ends in a final node $n_{\ell}$ of $H$. Then the corresponding state $\left(n_{\ell}, \sigma\right)$ reached in $\mathcal{A}(H)$ is final as $\sigma$ is satisfiable by Proposition 4. Conversely, if $\mathcal{A}(G)$ has an accepting sequence $\left(n_{0}, \sigma_{0}\right), \cdots\left(n_{l}, \sigma_{\ell}\right)$, but $n_{0} \cdots n_{\ell}$ is not $\left(Y, Y^{\prime}\right)$-drift-bounded, then, $\Phi_{Y, Y^{\prime}}(\rho)$ and $P F_{Y, Y^{\prime}}(\rho)$ have no solution. Again, by Lemma 1 , we have a contradiction, and $\mathcal{A}(H)$ accepts exactly the set of final $\left(Y, Y^{\prime}\right)$-drift-bounded paths of $H$. As a consequence, if $H$ is $\left(Y, Y^{\prime}\right)$-drift-bounded, then $\mathcal{L}(\mathcal{A}(H))=\emptyset$ iff $\mathcal{L}(H)=\emptyset$.

Proof (of Theorem 1). As every path of $\widehat{G}$ is $(K, \widehat{K})$-drift-bounded, in Proposition 5, taking $H=\widehat{G}, Y=K, Y^{\prime}=\widehat{K}$ implies $\mathcal{L}(\mathcal{A}(\widehat{G})) \neq \emptyset$ iff $\mathcal{L}(\widehat{G}) \neq \emptyset$ (iff 
$\mathcal{L}(G) \neq \emptyset$ by Corollary 1$)$. Now, checking that $\mathcal{L}(\mathcal{A}(\widehat{G})) \neq \emptyset$ is decidable in space logarithmic in $|G|, K$ and polynomial in $|\mathcal{P}|$.

The complexity follows since all states of $\mathcal{A}(H)$ are of the form $(n, \sigma)$ where $n$ is a node of $H$ and $\sigma$ a profile obtained inductively by application of function $\theta$. Every profile encodes a set of $|\mathcal{P}|^{2}$ inequations of the form $x_{e}-x_{f} \leq c_{e, f}$ where $c_{e, f}$ is an integer ranging between $-Y^{\prime}$ and $Y^{\prime}$ (since $Y \leq Y^{\prime}$ ). During Fourier-Motzkin elimination, we only use min and max over existing constants $c_{e, f}$ and $Y,-Y$. Also, the number of equations never grows above than $m^{2}$, as we simplify the system on the fly, where $m^{2}$ is the number of variables at the begining of Fourier-Motzkin elimination, that is $|T|+|\mathcal{P}|$, for $|T|$ the number of events in the TC-MSC $T$ that labels the node extending the current profiles. Thus, each application of Fourier-Motzkin elimination in our case is polynomial (unlike the general setting, which is doubly-exponential) in the size of input and so, the overall complexity of building on-the-fly the automaton $\mathcal{A}(H)$ and checking whether its language is empty is in PSPACE.

\section{Checking $K$-Drift-Boundedness of TC-MSC Graphs}

An automaton for minimal witnesses We search for a minimal witness path $\rho \cdot n=n_{0} \cdots n_{\ell} \cdot n$ in $\widehat{G}$ using an automaton $\mathcal{B}(\widehat{G})$. The first component of a state of $\mathcal{B}(\widehat{G})$ keeps track of the current node $n$. The second component will test for $(K, \widehat{K})$-drift-boundedness, which needs to hold for $\rho$ but not for $\rho \cdot n$. This is done by keeping track of a $\widehat{K}$-bounded profile. The last component keeps track of $P F_{K_{2}, K_{2}}(\rho)$ which is sufficient to check consistency of $\rho$ according to Proposition 6 . Theorem 2 is obtained using the following proposition (where $|\widehat{G}|$ $=$ no. of nodes of $\widehat{G}=$ no. of nodes of $G$ ):

Proposition 7. Let $G$ be a TC-MSC graph, and $\widehat{G}$ the associated full TC-MSC graph. Then there exists an automaton $\mathcal{B}(\widehat{G})$ such that $\mathcal{L}(\mathcal{B}(\widehat{G}))=\emptyset$ iff $\widehat{G}$ is $(K, \widehat{K})$-drift-bounded. Further, $\mathcal{B}(\widehat{G})$ has at most $|\widehat{G}| \times(2 \widehat{K}+1)^{|\mathcal{P}|^{2}} \times\left(2 K_{2}+1\right)^{|\mathcal{P}|^{2}}$ states, where $K_{2}=(|\mathcal{P}| / 2+1) \cdot \widehat{K}+D(\widehat{G})$.

Proof. The automaton $\mathcal{B}(\widehat{G})$ is defined as follows:

- The states of $\mathcal{B}(\widehat{G})$ are triples $(n, \sigma, \tau)$, with $n$ a node of $\widehat{G}, \sigma$ a $\widehat{K}$-bounded profile of $\widehat{G}$, and $\tau$ a $K_{2}$-bounded profile of $\widehat{G}$,

- The initial state of $\mathcal{B}(\widehat{G})$ is $\left(n_{i n}, P F_{K, \widehat{K}}\left(n_{i n}\right), P F_{K_{2}, K_{2}}\left(n_{i n}\right)\right)$.

- A state $(n, \sigma, \tau)$ of $\mathcal{B}(\widehat{G})$ is final if $\sigma$ is not satisfiable, but $\tau$ is. (Note that there is no condition on $n$ being final).

- There is a transition from $(n, \sigma, \tau)$ to $\left(n^{\prime}, \sigma^{\prime}, \tau^{\prime}\right)$ labeled by $n^{\prime}$ iff

1. $\widehat{G}$ contains a transition $n \rightarrow n^{\prime}$, and

2. $\sigma^{\prime}=\theta_{K, \widehat{K}}^{n \rightarrow n^{\prime}}(\sigma)$, and

3. $\tau^{\prime}=\theta_{K_{2}, K_{2}}^{n \rightarrow n^{\prime}}(\tau)$, and 
4. both $\sigma$ and $\tau^{\prime}$ are satisfiable.

Here $\sigma$ is satisfiable implies $\tau$ is satisfiable as $K_{2} \geq \widehat{K} \geq K$ and $\tau^{\prime}$ is satisfiable also implies that $\tau$ is. Thus condition 4 . can be replaced by $\sigma, \tau, \tau^{\prime}$ are satisfiable. However, note crucially that $\sigma^{\prime}$ is not required to be satisfiable.

We claim that $\mathcal{L}(\mathcal{B}(\widehat{G}))$ accepts exactly the set of minimal witnesses of $\widehat{G}$. In one direction, suppose that $\mathcal{L}(\mathcal{B}(\widehat{G}))$ is not empty. It means that there exists a sequence of nodes $\left(n_{0}, \sigma_{0}, \tau_{0}\right) \ldots\left(n_{k}, \sigma_{k}, \tau_{k}\right)$ such that $\left(n_{k}, \sigma_{k}, \tau_{k}\right)$ is final. Then, $\rho=n_{0} \cdots n_{k}$ is a path of $\widehat{G}, \sigma_{k}$ is not satisfiable, and $\tau_{k}$ is satisfiable. As $\tau_{k}$ is satisfiable, $\rho$ is consistent. As $\sigma_{k}$ is not satisfiable, it means that $\rho$ is not $(K, \widehat{K})$-drift-bounded. Lastly, as the transition $\left(n_{k-1}, \sigma_{k-1}, \tau_{k-1}\right) \rightarrow\left(n_{k}, \sigma_{k}, \tau_{k}\right)$ happened, it means that $\tau_{k-1}$ is satisfiable which means that $n_{0} \cdots n_{k-1}$ is $(K, \widehat{K})$-drift-bounded. Thus, the run defines a minimal witness of $\widehat{G}$.

Conversely, it is easy to see that $\mathcal{B}(\widehat{G})$ generates at least every path $\rho \cdot n$ with $\rho$ is $(K, \widehat{K})$-drift-bounded and $\rho \cdot n$ is consistent (due to the fact that $\sigma$ associated with $\rho$ is satisfiable, and using Proposition $5, \tau^{\prime}$ associated to $\rho \cdot n$ is also satisfiable). Hence if $\mathcal{L}(\mathcal{B}(\widehat{G}))$ is empty, no reachable state $s=(n, \sigma, \tau)$ is such that $\sigma$ not satisfiable and $\tau$ satisfiable, so in particular, no path with $\rho$ is $(K, \widehat{K})$-drift-bounded, $\rho \cdot n$ is consistent and $\rho \cdot n$ is not $(K, \widehat{K})$-drift-bounded has been found.

For the complexity, it suffices to remark that $Y$-bounded profiles have inequalities with constants lying between $-Y$ and $Y$ and then we get the maximal size of $\mathcal{B}(\widehat{G})$ as in Proposition 5 . 